\title{
Fluid dynamics in the warp drive spacetime geometry
}

\author{
Osvaldo L. Santos-Pereira ${ }^{1, a}$, Everton M. C. Abreu ${ }^{2,3,4, b}$, Marcelo B. Ribeiro ${ }^{1,4,5, c}$ (B) \\ ${ }^{1}$ Physics Institute, Universidade Federal do Rio de Janeiro, Rio de Janeiro, Brazil \\ 2 Physics Department, Universidade Federal Rural do Rio de Janeiro, Seropédica, Brazil \\ ${ }^{3}$ Physics Department, Universidade Federal de Juiz de Fora, Juiz de Fora, Brazil \\ ${ }^{4}$ Graduate Program in Applied Physics, Physics Institute, Universidade Federal do Rio de Janeiro, Rio de Janeiro, Brazil \\ ${ }^{5}$ Valongo Observatory, Universidade Federal do Rio de Janeiro, Rio de Janeiro, Brazil
}

Received: 14 September 2020 / Accepted: 26 January 2021 / Published online: 6 February 2021

(C) The Author(s) 2021

\begin{abstract}
The Alcubierre warp drive metric is a spacetime geometry featuring a spacetime distortion, called a warp bubble, where a massive particle inside it acquires global superluminal velocities, or warp speeds. This work presents solutions of the Einstein equations for the Alcubierre metric having fluid matter as gravity source. The energy-momentum tensor considered has two fluid contents, the perfect fluid and the parametrized perfect fluid (PPF), a tentative more flexible model whose aim is to explore the possibilities of warp drive solutions with positive matter density content. Santos-Pereira et al. (Eur Phys J C 80:786, 2020) already showed that the Alcubierre metric having dust as source connects this geometry to the Burgers equation, which describes shock waves moving through an inviscid fluid, but led the solutions back to vacuum. The same happened for two out of four solutions subcases for the perfect fluid. Other solutions for the perfect fluid indicate the possibility of warp drive with positive matter density, but at the cost of a complex solution for the warp drive regulating function. Regarding the PPF, solutions were also obtained indicating that warp speeds could be created with positive matter density. Weak, dominant, strong and null energy conditions were calculated for all studied subcases, being satisfied for the perfect fluid and creating constraints in the PPF quantities such that a positive matter density is also possible for creating a warp bubble. Summing up all results, energy-momentum tensors describing more complex forms of matter or field distributions generate solutions for the Einstein equations with the warp drive metric where a negative matter density might not be a strict precondition for attaining warp speeds.
\end{abstract}

\footnotetext{
a e-mail: olsp@if.ufrj.br

be-mail: evertonabreu@ufrrj.br

c e-mail: mbr@if.ufrj.br (corresponding author)
}

\section{Introduction}

It is well known that in general relativity particles can travel globally at superluminal speeds, whereas locally they cannot surpass the light speed. The warp drive spacetime geometry advanced by Alcubierre [1] uses these physical properties to propel material particles at superluminal speeds. It creates a limited spacetime distortion, called warp bubble, such that the spacetime is contracted in front of it and expanded behind the bubble as it moves along a geodesic. This warp drive metric is such that a particle trapped inside this bubble would locally move at subluminal speeds, whereas the bubble with the particle inside acquires global superluminal velocities, or warp speeds. In a seminal paper, Alcubierre also concluded that the warp metric would imply the violation of the energy conditions since it appeared that a negative energy density would be required for the creation of the warp bubble.

Since this original work many authors have contributed to our understanding of the theoretical details of the Alcubierre warp drive metric and the feasibility of matter particles acquiring warp speeds. Ford and Roman [2] applied quantum inequalities to calculate the amount of negative energy required to transport particles at superluminal speeds. They concluded that such energy requirements would be huge, so the amount of negative energy density necessary for the practical construction of a warp bubble would be impossible to achieve. Pfenning and Ford [3] also used quantum inequalities to calculate the necessary bubble parameters and energy for the warp drive viability, reaching an enormous amount of energy, ten orders of magnitude greater than the mass-energy of the entire visible universe, also being negative. Hiscock [4] computed the vacuum energy-momentum tensor (EMT) of a reduced two-dimensional quantized scalar field of the warp drive spacetime. He showed that in this reduced context that the EMT diverges if the apparent velocity of the bubble is greater than the speed of light. Such a divergence 
is connected to the construction of an horizon in this twodimensional spacetime. Due to the semiclassical effects, the superluminal travel via warp drive might be unfeasible. For example, to an observer within the warp drive bubble, the backward and forward walls look like the horizon of a white hole and of a black hole, respectively, resulting from Hawking radiation.

The issue of superluminal speeds of massive particles traveling faster than photons has also been studied by Krasnikov [5], who argued that this would not be possible if some conjectures for globally hyperbolic spacetimes are made. He described some spacetime topologies and their respective need of the tachyon existence for the occurrence of travel at warp speeds. This author also advanced a peculiar spacetime where superluminal travel would be possible without tachyons, named the Krasnikov tube by Everett and Roman [6], who generalized the metric designed by Krasnikov by proposing a tube in the direction of the particle's path providing a connection between Earth and a distant star. Inside the tube the spacetime is flat and the lightcones are opened out in order to allow for one direction superluminal travel. For the Krasnikov tube to work they showed that huge quantities of negative energy density would also be necessary. Since the tube does not possess closed timelike curves, it would be theoretically possible to design a two way non-overlapping tube system such that it would work as a time machine. In addition, the EMT is positive in some regions. Both the metric and the obtained EMT were thoroughly analyzed in Refs. $[7,8]$.

A relevant contribution to warp drive theory was made by van de Broeck [9], who demonstrated that a small modification of the original Alcubierre geometry greatly diminishes to a few solar masses the total negative energy necessary for the creation of the warp bubble distortion, a result that led him to hypothesize that other geometrical modifications of this type could further reduce in a dramatic fashion the amount of energy necessary to create a warp drive bubble. Natario [10] designed a new warp drive with zero expansion by using spherical coordinates and the $X$-axis as the polar one. Lobo and Visser [11,12] discussed that the center of the warp bubble, as proposed by Alcubierre, needs to be massless (see also [13,14]). A linearized model for both approaches was introduced and it was demonstrated that for small speeds the amassed negative energy inside the warp field is a robust fraction of the particle's mass inside the center of the warp bubble. Lee and Cleaver $[15,16]$ have looked at how external radiation might affect the Alcubierre warp bubble, possibly making it energetically unsustainable, and how a proposed warp field interferometer could not detect spacetime distortions. Mattingly et al. [17,18] discussed curvature invariants in the Natario and Alcubierre warp drives.

In a previous paper [19] we have considered some of these issues, but from a different angle. The Alcubierre metric was not advanced as a solution of the Einstein equations, as it was originally proposed simply as an ad hoc geometry designed to create a spacetime distortion such that a massive particle inside it travels at warp speeds, whereas locally it never exceeds the light speed. The basic question was then which possible types of matter-energy sources are capable of creating a warp bubble. To answer this question the Einstein equations have to be solved with some form of EMT as a source. The simplest one to start with is incoherent matter. Following this line of investigation, we showed that the dust solutions of the Einstein equations for the warp drive metric implied in vacuum, that is, such a distribution is incapable of creating a warp bubble; nevertheless, the Burgers equation appeared as part of the solution of the Einstein equations. In addition, since the Burgers equation describes shock waves moving in an inviscid fluid, it was also found that these shock waves behave as plane waves [24-28].

In this paper we generalize the results obtained in Ref. [19] by following the next logical step, that is, investigating the perfect fluid as EMT source for the Alcubierre metric. We also propose a slightly generalized perfect fluid EMT, called here the parametrized perfect fluid (PPF), in order to produce a tentatively more flexible model such that the pressure may have different parameter values. The aim is to see if more flexible EMT distributions could relax the original requirement that warp speeds could only be achieved by means of a negative matter density.

For the perfect fluid EMT solutions we found that two out of four subcases turn out to be the dust solution of Ref. [19] where both the matter density and the pressure vanish, but the Burgers equation also appears as a result of the solutions of the Einstein equations [29-31]. Two other subcases, however, indicate that warp speeds are possible with positive matter density, but at the cost of a complex solution for the warp metric regulating function. Weak, dominant, strong and null energy conditions were calculate for both EMTs and all perfect fluid solutions satisfy them. In the case of the PPF, two out of four solutions give rise to a nonlinear equation of state linking various pressures to the matter density. Other solutions produced results where a nonvanishing pressure occurs with a vanishing matter density, a condition considered unphysical and then dismissed. The solutions also produced parameters and equations of state related to pressure and inequalities that satisfy all the energy conditions. These results indicate that energy-momentum tensors describing more complex forms of matter distributions generate solutions for the Einstein equations with the warp drive metric where negative matter density might not be a strict precondition.

The plan for the paper is as follows. In Sect. 2 we briefly review the Alcubierre warp drive theory and present the relevant equations and all nonzero components of the Einstein tensor for the warp drive metric. In Sect. 3 the Einstein equa- 
tions are then solved and solutions presented for the warp drive metric having a perfect fluid gravity source. In Sect. 4 the nonzero components of the Einstein tensor in the warp drive geometry are written in terms of the PPF EMT. Solutions for this more flexible EMT are also obtained and studied in all subcases. Section 5 presents the EMT divergence of both the perfect fluid and the PPF, whereas Sect. 6 discusses the energy conditions for the two types of EMTs. Section 7 provides further discussions on the results presented in the previous sections, and Sect. 8 presents our conclusions.

\section{Einstein equations}

We shall start this section by briefly reviewing the Alcubierre warp drive metric. Subsequently, the nonzero components of the Einstein tensor of this metric will also be explicitly shown. The expressions presented in this section form the basic set of equations required for the next sections.

\subsection{The Alcubierre warp drive geometry}

The geometry advanced in Ref. [1] may be written as follows:

$\mathrm{d} s^{2}=-\left(\alpha^{2}-\beta_{i} \beta^{i}\right) \mathrm{d} t^{2}+2 \beta_{i} \mathrm{~d} x^{i} \mathrm{~d} t+\gamma_{i j} \mathrm{~d} x^{i} \mathrm{~d} x^{j}$,

where $d \tau$ is the proper time lapse, $\alpha$ is the lapse function, $\beta^{i}$ is the spacelike shift vector and $\gamma_{i j}$ is the spatial metric for the hypersurfaces. ${ }^{1}$ The lapse function $\alpha$ and the shift vector $\beta^{i}$ are functions to be determined, whereas $\gamma_{i j}$ is a positivedefinite metric on each of the spacelike hypersurfaces, for all values of time, a feature that makes the spacetime globally hyperbolic $[21,22]$.

Alcubierre [1] assumed the following particular parameter choices for Eq. (2.1):

$$
\begin{aligned}
\alpha & =1, \\
\beta^{1} & =-v_{s}(t) f\left[r_{s}(t)\right], \\
\beta^{2} & =\beta^{3}=0, \\
\gamma_{i j} & =\delta_{i j} .
\end{aligned}
$$

Hence, the Alcubierre warp drive metric is given by

$$
\begin{aligned}
\mathrm{d} s^{2}=- & {\left[1-v_{s}(t)^{2} f\left(r_{s}\right)^{2}\right] \mathrm{d} t^{2}-v_{s}(t) f\left(r_{s}\right) \mathrm{d} x \mathrm{~d} t } \\
& +\mathrm{d} x^{2}+\mathrm{d} y^{2}+\mathrm{d} z^{2}
\end{aligned}
$$

1 Throughout this paper Greek indices will range from 0 to 3, whereas the Latin ones indicate the spacelike hypersurfaces and will range from 1 to 3 . where $v_{s}(t)$ is the velocity of the center of the bubble moving along the curve $x_{S}(t)$. This is given by the following expression:

$v_{s}(t)=\frac{\mathrm{d} x_{s}(t)}{\mathrm{d} t}$.

The function $f\left(r_{s}\right)$ is the warp drive regulating function. It describes the shape of the warp bubble, which is given by the following expression [1]:

$f\left(r_{s}\right)=\frac{\tanh \left[\sigma\left(r_{s}+R\right)\right]-\tanh \left[\sigma\left(r_{s}-R\right)\right]}{2 \tanh (\sigma R)}$,

where $\sigma$ and $R$ are parameters to be determined. The variable $r_{s}(t)$ defines the distance from the center of the bubble $\left[x_{S}(t), 0,0\right]$ to a generic point $(x, y, z)$ on the surface of the bubble, given by the following equation:

$r_{s}(t)=\sqrt{\left[x-x_{s}(t)\right]^{2}+y^{2}+z^{2}}$.

From the above one can see that the motion is onedimensional, since the $x$-coordinate is the only one perturbed by the function $x_{S}(t)$.

\subsection{Einstein tensor components}

Let us now adopt Alcubierre's original notation by assuming

$\beta=-\beta^{1}=v_{s}(t) f\left(r_{s}\right)$

in Eq. (2.3). Then the nonzero components of the Einstein tensor for the metric (2.6) are given by the following expressions:

$$
\begin{aligned}
G_{00}= & -\frac{1}{4}\left(1+3 \beta^{2}\right)\left[\left(\frac{\partial \beta}{\partial y}\right)^{2}+\left(\frac{\partial \beta}{\partial z}\right)^{2}\right] \\
& -\beta\left(\frac{\partial^{2} \beta}{\partial y^{2}}+\frac{\partial^{2} \beta}{\partial z^{2}}\right), \\
G_{01}= & \frac{3}{4}\left[\left(\frac{\partial \beta}{\partial y}\right)^{2}+\left(\frac{\partial \beta}{\partial z}\right)^{2}\right] \\
& +\frac{1}{2}\left(\frac{\partial^{2} \beta}{\partial y^{2}}+\frac{\partial^{2} \beta}{\partial z^{2}}\right), \\
G_{02}= & -\frac{1}{2} \frac{\partial^{2} \beta}{\partial x \partial y} \\
& -\frac{\beta}{2}\left(2 \frac{\partial \beta}{\partial y} \frac{\partial \beta}{\partial x}+\beta \frac{\partial^{2} \beta}{\partial x \partial y}+\frac{\partial^{2} \beta}{\partial t \partial y}\right), \\
G_{03}= & -\frac{1}{2} \frac{\partial^{2} \beta}{\partial x \partial z} \\
& -\frac{\beta}{2}\left(2 \frac{\partial \beta}{\partial z} \frac{\partial \beta}{\partial x}+\beta \frac{\partial^{2} \beta}{\partial x \partial z}+\frac{\partial^{2} \beta}{\partial t \partial z}\right),
\end{aligned}
$$




$$
\begin{aligned}
G_{11}= & -\frac{3}{4}\left[\left(\frac{\partial \beta}{\partial y}\right)^{2}+\left(\frac{\partial \beta}{\partial z}\right)^{2}\right], \\
G_{12}= & \frac{1}{2}\left(2 \frac{\partial \beta}{\partial y} \frac{\partial \beta}{\partial x}+\beta \frac{\partial^{2} \beta}{\partial x \partial y}+\frac{\partial^{2} \beta}{\partial t \partial y}\right), \\
G_{13}= & \frac{1}{2}\left(2 \frac{\partial \beta}{\partial z} \frac{\partial \beta}{\partial x}+\beta \frac{\partial^{2} \beta}{\partial x \partial z}+\frac{\partial^{2} \beta}{\partial t \partial z}\right), \\
G_{23}= & \frac{1}{2} \frac{\partial \beta}{\partial z} \frac{\partial \beta}{\partial y}, \\
G_{22}= & -\left[\frac{\partial^{2} \beta}{\partial t \partial x}+\beta \frac{\partial^{2} \beta}{\partial x^{2}}+\left(\frac{\partial \beta}{\partial x}\right)^{2}\right] \\
& -\frac{1}{4}\left[\left(\frac{\partial \beta}{\partial y}\right)^{2}-\left(\frac{\partial \beta}{\partial z}\right)^{2}\right], \\
G_{33}= & -\left[\frac{\partial^{2} \beta}{\partial t \partial x}+\beta \frac{\partial^{2} \beta}{\partial x^{2}}+\left(\frac{\partial \beta}{\partial x}\right)^{2}\right] \\
& +\frac{1}{4}\left[\left(\frac{\partial \beta}{\partial y}\right)^{2}-\left(\frac{\partial \beta}{\partial z}\right)^{2}\right] .
\end{aligned}
$$

\section{Perfect fluid}

Besides incoherent matter, or dust, already studied in Ref. [19], the simplest matter-energy distribution to be considered as a gravity source for the possible creation of a warp bubble, and then warp speeds, is the perfect fluid. Hence, this section will discuss matter content solutions of the Einstein equations considering a perfect fluid matter source EMT for the Alcubierre metric.

\subsection{Perfect fluid content solutions}

The EMT for a perfect fluid may be written as follows:

$$
T_{\alpha \beta}=(\mu+p) u_{\alpha} u_{\beta}+p g_{\alpha \beta},
$$

where $\mu$ is the matter density, $p$ is the fluid pressure, $g_{\alpha \beta}$ is the metric tensor and $u_{\alpha}$ is the four-velocity of an observer inside the fluid. Perfect fluids have no shear stress, rotation, heat conduction or viscosity, nevertheless this ideal fluid provides a more complex matter content than simple dust [20], allowing us to study if a warp bubble can be created with this gravity source and how the respective gravity field equations solutions can be understood.

For the metric (2.6) the perfect fluid EMT assumes the following form:

$T_{\alpha \beta}=\left(\begin{array}{cccc}\mu+\beta^{2} p & -\beta p & 0 & 0 \\ -\beta p & p & 0 & 0 \\ 0 & 0 & p & 0 \\ 0 & 0 & 0 & p\end{array}\right)$

Let us now use Eqs. (2.11)-(2.20) with the EMT above in the Einstein equations. Substituting components $G_{11}=8 \pi T_{11}$ and $G_{01}=8 \pi T_{01}$ into $G_{00}=8 \pi T_{00}$, after some algebra and simplifications we may write the following expression:

$T_{00}+2 \beta T_{01}+\frac{1}{3}\left(3 \beta^{2}-1 x\right) T_{11}=0$.

Substituting the values for the EMT components $T_{00}=\mu+$ $\beta^{2} p, T_{01}=-\beta p, T_{11}=p$ Eq. (3.3) results in the following expression:

$p=3 \mu$.

This is an equation of state for the Alcubierre metric having a perfect fluid gravity source EMT.

The component $G_{23}$ is zero since $T_{23}=0$. This case leads to either $\partial \beta / \partial y$, or $\partial \beta / \partial z$, or both, equal to zero. Let us now analyze these possibilities and its consequences.

Case 1: $\left[\frac{\partial \boldsymbol{\beta}}{\partial z}=\mathbf{0}\right]$ As $\beta$ does not depend on $z$, the Einstein tensor components $G_{13}, G_{23}$ and $G_{03}$ are identically zero. Substituting this case into $G_{11}=8 \pi T_{11}$, where $G_{11}$ is given by Eq. (2.15) and $T_{11}=p$, there follows immediately the result

$$
\frac{3}{4}\left(\frac{\partial \beta}{\partial y}\right)^{2}=-8 \pi p
$$

Substituting $(\partial \beta / \partial y)^{2}$ above into $G_{01}=8 \pi T_{01}$, where $G_{01}$ is given by Eq. (2.12) and $T_{01}=-\beta p$ from Eq. (3.2), as well as $G_{12}=8 \pi T_{12}$ and $G_{02}=$ $8 \pi T_{02}$, where $T_{12}=0$ and $T_{02}=0$, the Einstein equations are reduced to the following equations:

$$
\begin{aligned}
& p=3 \mu, \\
& \left(\frac{\partial \beta}{\partial y}\right)^{2}=-\frac{32}{3} \pi p=-32 \pi \mu, \\
& \frac{\partial^{2} \beta}{\partial y^{2}}=0, \\
& \frac{\partial^{2} \beta}{\partial x \partial y}=0, \\
& 2 \frac{\partial \beta}{\partial y} \frac{\partial \beta}{\partial x}+\frac{\partial^{2} \beta}{\partial t \partial y}=0, \\
& \frac{\partial^{2} \beta}{\partial t \partial x}+\beta \frac{\partial^{2} \beta}{\partial x^{2}}+\left(\frac{\partial \beta}{\partial x}\right)^{2} \\
& =-\frac{64}{3} \pi p=-64 \pi \mu, \\
& \frac{\partial^{2} \beta}{\partial t \partial x}+\beta \frac{\partial^{2} \beta}{\partial x^{2}}+\left(\frac{\partial \beta}{\partial x}\right)^{2}
\end{aligned}
$$




$$
=-\frac{128}{3} \pi p=-128 \pi \mu .
$$

Equation (3.7) implies that $\partial \beta / \partial y$ must be constant, since the pressure $p$ is assumed constant. Equation (3.8) also shows that $\beta$ must be a linear function of the $y$-coordinate, which means that $\beta$ must have a possible additional dependence of arbitrary functions on $t$ and $x$. The two expressions in Eqs. (3.11) and (3.12) constitute the same homogeneous partial differential equation, but with different inhomogeneous parts, so the solution of the inhomogeneous equation is not unique, unless the pressure $p$ is zero. Then, considering these points and Eqs. (3.7) and (3.10), it follows that

$$
\frac{\partial \beta}{\partial y} \frac{\partial \beta}{\partial x}=0
$$

which means that either of these partial derivatives, or both, vanish. Let us discuss both possibilities.

Case 1a: $\left[\frac{\partial \boldsymbol{\beta}}{\partial z}=\mathbf{0}\right.$ and $\left.\frac{\partial \boldsymbol{\beta}}{\partial \boldsymbol{x}}=\mathbf{0}\right]$ For this case the set of partial differential equations from Eqs. (3.6) to (3.12) simplify to

$$
\begin{aligned}
& p=3 \mu, \\
& \frac{\partial \beta}{\partial y}= \pm \sqrt{-32 \pi \mu} .
\end{aligned}
$$

The above equations mean that the matter density $\mu$ must be negative or zero for a non-complex solution of the Einstein equations, and $\beta$ must be a function of only the two coordinates $t$ and $x$. The equation above is readily integrated, yielding

$$
\beta(t, y)= \pm \sqrt{-32 \pi \mu} y+g(t)
$$

where $g(t)$ is a function to be determined by the boundary conditions.

Case $1 \mathrm{~b}:\left[\frac{\partial \boldsymbol{\beta}}{\partial z}=\mathbf{0}\right.$ and $\left.\frac{\partial \boldsymbol{\beta}}{\partial \boldsymbol{y}}=\mathbf{0}\right]$ In this case the pressures vanishes, since $\partial \beta / \partial y=0$, and the set of partial differential equations (3.6) to (3.12) simplify to,

$p=3 \mu=0$,

$\frac{\partial^{2} \beta}{\partial t \partial x}+\beta \frac{\partial^{2} \beta}{\partial x^{2}}+\left(\frac{\partial \beta}{\partial x}\right)^{2}=0$

Therefore, for $p=0$ the equation of state $p=3 \mu$ implies zero matter density as well, which reduces the solution to the dust case and then vacuum. This also leads to the appearance of shock waves as plane waves, since $\beta=\beta(x, t)$ and the field equations are reduced to the Burgers equation, as studied in Ref. [19]. This is the case because Eq. (3.18) can be written in the following form:

$$
\frac{\partial \beta}{\partial t}+\frac{1}{2} \frac{\partial}{\partial x}\left(\beta^{2}\right)=h(t)
$$

Here $h(t)$ is a generic function to be determined by boundary conditions. In its homogeneous form, where $h(t)=0$, it takes the conservation form of the inviscid Burgers equation,

$$
\frac{\partial \beta}{\partial t}+\frac{1}{2} \frac{\partial}{\partial x}\left(\beta^{2}\right)=0
$$

See Ref. [19] for details of the Burgers equation in this context.

Case 2: $\left[\frac{\partial \boldsymbol{\beta}}{\partial \boldsymbol{y}}=\mathbf{0}\right]$ As $\beta$ does not depend on the $y$ coordinate, it is easy to see that $G_{12}, G_{23}$ and $G_{02}$ are identically zero. In addition, considering this case with $G_{11}=8 \pi T_{11}$, it follows immediately that

$$
-\frac{3}{4}\left(\frac{\partial \beta}{\partial z}\right)^{2}=8 \pi p
$$

Substituting Eq. (3.21) in $G_{01}=8 \pi T_{01}$, where $G_{01}$ is given by Eq. (2.12), $T_{01}=-\beta p$ from Eq. (3.2), and inserting the component $G_{13}=8 \pi T_{13}$ into the component $G_{03}=8 \pi T_{03}$, where $T_{13}=T_{03}=0$, after some algebra we obtain the following expressions:

$p=3 \mu$,

$\left(\frac{\partial \beta}{\partial z}\right)^{2}=-\frac{32}{3} \pi p$

$\frac{\partial^{2} \beta}{\partial z^{2}}=0$,

$\frac{\partial^{2} \beta}{\partial x \partial z}=0$,

$2 \frac{\partial \beta}{\partial z} \frac{\partial \beta}{\partial x}+\frac{\partial^{2} \beta}{\partial t \partial z}=0$,

$\frac{\partial^{2} \beta}{\partial t \partial x}+\beta \frac{\partial^{2} \beta}{\partial x^{2}}+\left(\frac{\partial \beta}{\partial x}\right)^{2}=-\frac{64}{3} \pi p$,

$\frac{\partial^{2} \beta}{\partial t \partial x}+\beta \frac{\partial^{2} \beta}{\partial x^{2}}+\left(\frac{\partial \beta}{\partial x}\right)^{2}=-\frac{128}{3} \pi p$.

Equation (3.24) shows that $\beta$ is a linear function with respect to the $z$-coordinate. Equation (3.23) implies that $\partial \beta / \partial z$ must be constant since the pressure $p$ is assumed to be a constant. This means that all second partial derivatives of $\partial \beta / \partial z$ must vanish. 
Equations (3.27) and (3.28) are the same homogeneous partial differential equation, but their right hand sides are different. Hence, the solution of the non-homogeneous equation is not unique, unless the pressure $p$ is zero. Considering Eq. (3.26), this case also unfolds in two possibilities, since

$$
\frac{\partial \beta}{\partial z} \frac{\partial \beta}{\partial x}=0
$$

and either or both are zero. Let us now analyze each

Case 2a: $\left[\frac{\partial \boldsymbol{\beta}}{\partial y}=\mathbf{0}\right.$ and $\left.\frac{\partial \boldsymbol{\beta}}{\partial x}=\mathbf{0}\right]$ For this case Eqs. (3.22) to (3.28) yield

$$
\begin{aligned}
& p=3 \mu \\
& \frac{\partial \beta}{\partial z}= \pm \sqrt{-32 \pi \mu} \\
& \frac{\partial \beta}{\partial z}= \pm \sqrt{ \pm 96 \pi \mu}
\end{aligned}
$$

Equation (3.31) means that the matter density $\mu$ must be negative or zero for a non-complex solution of the Einstein equations. Equation (3.32) allows for a possible positive matter density. In addition, $\beta$ has its dependence reduced to $\beta=\beta(z, t)$. If the matter density $\mu$ is assumed constant, then the above expressions can be integrated, yielding

$$
\begin{aligned}
& \beta(z, t)= \pm \sqrt{-32 \pi \mu} z+\bar{g}(t), \\
& \beta(z, t)= \pm \sqrt{ \pm 96 \pi \mu} z+\bar{h}(t),
\end{aligned}
$$

where $\bar{g}(t)$ and $\bar{h}(t)$ are arbitrary functions to be determined by boundary conditions.

Case $2 \mathrm{~b}:\left[\frac{\partial \beta}{\partial y}=\mathbf{0}\right.$ and $\left.\frac{\partial \beta}{\partial z}=\mathbf{0}\right]$ For this subcase Eq. (3.23) implies zero pressure, and the set of partial differential equations (3.22) to (3.28) simplify to

$$
\begin{aligned}
& p=3 \mu=0, \\
& \frac{\partial^{2} \beta}{\partial t \partial x}+\beta \frac{\partial^{2} \beta}{\partial x^{2}}+\left(\frac{\partial \beta}{\partial x}\right)^{2}=0,
\end{aligned}
$$

where the last equation is the result of the only nonzero Einstein tensor components, $G_{22}$ and $G_{33}$. These results are the same as Case $1 \mathrm{~b}$ above, that is, the dust solution for the Alcubierre warp drive metric that results in the Burgers equation (3.19) and its inviscid form given by Eq. (3.20), and shock waves as well as plane waves [19].
Table 1 summarizes all cases and their respective results of the Einstein equations with the Alcubierre warp drive metric having a perfect fluid matter content as gravity source.

\subsection{Discussion}

Cases $1 \mathrm{~b}$ and $2 \mathrm{~b}$ are simply the dust case already studied in Ref. [19], which apparently is unable to generate a warp bubble since this is a vacuum solution, although it connects the warp metric to the Burgers equation and then to shock waves in the form of plane waves.

Cases 1a and 2a share the equation of state $p=3 \mu$, but the coordinate dependencies are different, since $\beta=\beta(y, t)$ and $\beta=\beta(z, t)$, respectively. For $\beta$ to be a real valued function the matter density $\mu$ must be negative in Case 1a. From Eqs. (3.16) and (3.33) we have assumed a constant matter density, which means a straightforward integration, but $\mu$ can be also a function of the two coordinates $t$ and $y$ in Case 1a, or a function of the $t$ and $z$ coordinates in Case 2a.

Inasmuch as the matter density must be negative for real solutions, one could think of defining the total mass-energy density as follows:

$$
\begin{aligned}
& \mu\left(t, x^{j}\right)=\mu^{+}+a\left(t, x^{j}\right) \mu^{-} \leq 0, \\
& x^{j}=y, z,
\end{aligned}
$$

where $\mu^{+}$is the positive portion of the matter density of the perfect fluid and $\mu^{-}$its negative portion that would allow the warp bubble to exist. $a\left(t, x^{j}\right)$ would be a regulating function that depends on both time $t$ and space $x^{j}$ coordinates, being related to the shape and location of the bubble. Remembering that $x^{j}=y$ for Case $1 \mathrm{a}$ and $x^{j}=z$ for Case 2a, since $\mu\left(t, x^{j}\right)$ must be negative there would be a restriction for the positive and negative portions of the matter density in Eq. (3.37).

It might be argued that there is no problem in complex solutions for $\beta$, but in the warp drive scenario $\beta=v_{s}(t) f\left(r_{s}\right)$ determines both the velocity and the shape of the bubble, so either the velocity $v_{s}(t)$ or the regulating function $f\left(r_{s}\right)$ of the bubble shape must be complex. A complex velocity has no physical meaning, but a complex regulating function could be acceptable if we only consider its real part. Thus, in such a situation the formation of a warp bubble capable of generating warp speeds could still be possible in the presence of a perfect fluid positive matter density EMT as gravity source.

Nevertheless, caution is required here because if the result of integrating $\beta$ turns out to be purely imaginary it is not clear what the bubble shape being represented by an imaginary function means. Therefore, in principle it seems reasonable to start with $\beta$ being a real function and the warp bubble requiring a perfect fluid with negative mass-energy density 
Table 1 Summary of all solutions of the Einstein equations with the Alcubierre warp drive metric having perfect fluid EMT as mass-energy source

\begin{tabular}{|c|c|c|}
\hline Case & Condition & Results \\
\hline 1) $\frac{\partial \beta}{\partial z}=0$ & 1a) $\frac{\partial \beta}{\partial x}=0$ & $\begin{array}{l}p=3 \mu \\
\beta=\beta(y, t) \\
\frac{\partial \beta}{\partial y}= \pm \sqrt{-32 \pi \mu}\end{array}$ \\
\hline & 1b) $\frac{\partial \beta}{\partial y}=0$ & $\begin{array}{l}p=3 \mu=0 \\
\beta=\beta(x, t) \\
\frac{\partial \beta}{\partial t}+\frac{1}{2} \frac{\partial}{\partial x}\left(\beta^{2}\right)=h(t) \\
\rightarrow \text { this is the dust solution of Ref. [19] }\end{array}$ \\
\hline 2) $\frac{\partial \beta}{\partial y}=0$ & 2a) $\frac{\partial \beta}{\partial x}=0$ & $\begin{array}{l}p=3 \mu \\
\beta=\beta(z, t) \\
\frac{\partial \beta}{\partial z}= \pm \sqrt{-32 \pi \mu} \\
\frac{\partial \ddot{\beta}}{\partial z}= \pm \sqrt{ \pm 96 \pi \mu}\end{array}$ \\
\hline & $2 b) \frac{\partial \beta}{\partial z}=0$ & $\begin{array}{l}p=3 \mu=0 \\
\beta=\beta(x, t) \\
\frac{\partial \beta}{\partial t}+\frac{1}{2} \frac{\partial}{\partial x}\left(\beta^{2}\right)=h(t) \\
\rightarrow \text { this is the dust solution of Ref. [19] }\end{array}$ \\
\hline
\end{tabular}

for warp speeds to be physically viable. But it seems to us that this point remains open to debate.

Considering the results above it is apparent that a perfect fluid EMT generated a more complex set of solutions of the Einstein equations than the dust one, then it is conceivable that viable warp speeds are also possible in more complex EMTs. One such possibility will be discussed next.

\section{Parametrized perfect fluid}

Let us propose a generalization of the perfect fluid EMT having seven quantities, namely the mass-energy density $\mu$, the $\beta$ function and five different pressures $A, B, C, D$ and $p$. The last quantity $D$ is a momentum density parameter. In the perfect fluid of Eq. (3.2) the pressure denoted by $p$ is all the same in the EMT, a constraint that has been relaxed here. Let us call the perfect fluid generalization with the quantities above the parametrized perfect fluid (PPF). Its respective EMT may be written

$T_{\alpha \sigma}=\left(\begin{array}{cccc}\mu+\beta^{2} p & -\beta D & 0 & 0 \\ -\beta D & A & 0 & 0 \\ 0 & 0 & B & 0 \\ 0 & 0 & 0 & C\end{array}\right)$

The quantities $A, B, C, D$ and $p$ will not be assumed to be constants, but rather functions of the spacetime coordinates $(t, x, y, z)$.

This is clearly a more flexible EMT than the perfect fluid, and it is being proposed here as a tentative model in order to explore the consequences of more complex EMTs in terms of generating possible positive matter solutions of the Einstein equations with the warp drive metric without the caveats of the perfect fluid solutions discussed above. It is a tentative proposal for a more flexible, or toy, model for the possible creation of warp bubbles, and then warp speeds. Section 7.2 provides more details on the physics of this specific fluid proposal.

The nonzero components of the Einstein equations for the PPF are given by the following expressions:

$$
\begin{aligned}
&- \frac{1}{4}\left(1+3 \beta^{2}\right)\left[\left(\frac{\partial \beta}{\partial y}\right)^{2}+\left(\frac{\partial \beta}{\partial z}\right)^{2}\right] \\
&-\beta\left(\frac{\partial^{2} \beta}{\partial y^{2}}+\frac{\partial^{2} \beta}{\partial z^{2}}\right)=8 \pi\left(\mu+\beta^{2} p\right), \\
& \frac{3}{4} \beta\left[\left(\frac{\partial \beta}{\partial y}\right)^{2}+\left(\frac{\partial \beta}{\partial z}\right)^{2}\right]+\frac{1}{2}\left(\frac{\partial^{2} \beta}{\partial y^{2}}+\frac{\partial^{2} \beta}{\partial z^{2}}\right)=-8 \pi \beta D, \\
&-\frac{1}{2} \frac{\partial^{2} \beta}{\partial x \partial y}-\frac{\beta}{2}\left(2 \frac{\partial \beta}{\partial y} \frac{\partial \beta}{\partial x}\right. \\
&+\left.\beta \frac{\partial^{2} \beta}{\partial x \partial y}+\frac{\partial^{2} \beta}{\partial t \partial y}\right)=0, \\
&- \frac{1}{2} \frac{\partial^{2} \beta}{\partial x \partial z}-\frac{\beta}{2}\left(2 \frac{\partial \beta}{\partial z} \frac{\partial \beta}{\partial x}\right. \\
&+\left.\beta \frac{\partial^{2} \beta}{\partial x \partial z}+\frac{\partial^{2} \beta}{\partial t \partial z}\right)=0, \\
&- \frac{3}{4}\left[\left(\frac{\partial \beta}{\partial y}\right)^{2}+\left(\frac{\partial \beta}{\partial z}\right)^{2}\right]=8 \pi A \\
& \frac{1}{2}\left(2 \frac{\partial \beta}{\partial y} \frac{\partial \beta}{\partial x}+\beta \frac{\partial^{2} \beta}{\partial x \partial y}+\frac{\partial^{2} \beta}{\partial t \partial y}\right)=0,
\end{aligned}
$$




$$
\begin{aligned}
& \frac{1}{2}\left(2 \frac{\partial \beta}{\partial z} \frac{\partial \beta}{\partial x}+\beta \frac{\partial^{2} \beta}{\partial x \partial z}+\frac{\partial^{2} \beta}{\partial t \partial z}\right)=0, \\
& \frac{1}{2} \frac{\partial \beta}{\partial z} \frac{\partial \beta}{\partial y}=0, \\
& -\left[\frac{\partial^{2} \beta}{\partial t \partial x}+\beta \frac{\partial^{2} \beta}{\partial x^{2}}+\left(\frac{\partial \beta}{\partial x}\right)^{2}\right] \\
& -\frac{1}{4}\left[\left(\frac{\partial \beta}{\partial y}\right)^{2}-\left(\frac{\partial \beta}{\partial z}\right)^{2}\right]=8 \pi B, \\
& -\left[\frac{\partial^{2} \beta}{\partial t \partial x}+\beta \frac{\partial^{2} \beta}{\partial x^{2}}+\left(\frac{\partial \beta}{\partial x}\right)^{2}\right] \\
& +\frac{1}{4}\left[\left(\frac{\partial \beta}{\partial y}\right)^{2}-\left(\frac{\partial \beta}{\partial z}\right)^{2}\right]=8 \pi C .
\end{aligned}
$$

Substituting Eqs. (4.6) and (4.3) into Eq. (4.2) it follows immediately that

$\mu=\beta^{2}(2 D-A-p)+\frac{A}{3}$.

This expression shows that the fluid density not only depends on the pressure components $A$ and $p$, but also on the momentum component $D$ and the warp bubble, since it varies with the shift vector $\beta$ and, hence, the bubble movement. So, the bubble shape modifies the fluid density in a local way, a result that may imply an analogy with classical fluid dynamics and shock waves in fluids with global velocity greater than the speed of sound in that medium.

Applying this analogy to the warp drive, it may mean that the warp bubble plays the role of a shock wave in a fluid that moves with apparent velocity greater than the speed of light for an outside observer far away from the bubble, this being a result of the nonlinearity of the Einstein equations. The bubble modifies the fluid density, which then causes the bubble motion. This classical relativistic fluid analogy may be a physical argument for a mechanism which accounts for the great amount of energy necessary for the feasibility of the warp drive.

After some algebra on the set of Einstein equations above they can be rewritten

$$
\begin{aligned}
\beta^{2}(2 D-A-p)+\frac{A}{3} & =\mu \\
\frac{\partial^{2} \beta}{\partial x \partial y} & =0 \\
\frac{\partial^{2} \beta}{\partial x \partial z} & =0 \\
\left(\frac{\partial \beta}{\partial y}\right)^{2}+\left(\frac{\partial \beta}{\partial z}\right)^{2} & =-\frac{32}{3} \pi A, \\
\left(\frac{\partial \beta}{\partial y}\right)^{2}-\left(\frac{\partial \beta}{\partial z}\right)^{2} & =16 \pi(C-B),
\end{aligned}
$$

$$
\begin{aligned}
2 \frac{\partial \beta}{\partial y} \frac{\partial \beta}{\partial x}+\frac{\partial^{2} \beta}{\partial t \partial y} & =0, \\
2 \frac{\partial \beta}{\partial z} \frac{\partial \beta}{\partial x}+\frac{\partial^{2} \beta}{\partial t \partial z} & =0, \\
\frac{\partial \beta}{\partial z} \frac{\partial \beta}{\partial y} & =0 \\
\frac{\partial}{\partial x}\left[\frac{\partial \beta}{\partial t}+\frac{1}{2} \frac{\partial}{\partial x}\left(\beta^{2}\right)\right] & =-32 \pi(B+C), \\
\frac{\partial^{2} \beta}{\partial y^{2}}+\frac{\partial^{2} \beta}{\partial z^{2}} & =16 \pi \beta(A-D) .
\end{aligned}
$$

Equation (4.20) shows that the solutions for the set of differential equations above have similar alternative cases to the perfect fluid solutions, that is, either $\partial \beta / \partial z=0$ and/or $\partial \beta / \partial y=0$. Both situations and their respective subcases will be discussed next.

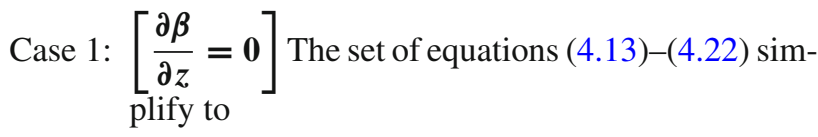

$$
\begin{aligned}
& \mu=\beta^{2}(2 D-A-p)+\frac{A}{3}, \\
& \frac{\partial^{2} \beta}{\partial x \partial y}=0, \\
& \left(\frac{\partial \beta}{\partial y}\right)^{2}=-\frac{32}{3} \pi A=16 \pi(C-B), \\
& 2 \frac{\partial \beta}{\partial y} \frac{\partial \beta}{\partial x}+\frac{\partial^{2} \beta}{\partial t \partial y}=0, \\
& \frac{\partial}{\partial x}\left[\frac{\partial \beta}{\partial t}+\frac{1}{2} \frac{\partial}{\partial x}\left(\beta^{2}\right)\right]=-32 \pi(B+C) . \\
& \frac{\partial^{2} \beta}{\partial y^{2}}=16 \pi \beta(A-D) .
\end{aligned}
$$

From Eq. (4.25) a relation between the pressures $A, B$ and $C$ is straightforward,

$B=C+\frac{2}{3} A$

From Eqs. (4.24) and (4.25) it is easy to verify that $A$ and $C-B$ do not depend on the $x$-coordinate. In addition, for real solutions $A$ must be negative, assuming only real values that are equal to or smaller than zero. Differentiating Eq. (4.25) with respect to $x$ yields

$$
2 \frac{\partial \beta}{\partial y} \frac{\partial^{2} \beta}{\partial x \partial y}=0 \Longrightarrow \frac{\partial \beta}{\partial y}=0 \text { and/or } \frac{\partial^{2} \beta}{\partial x \partial y}=0 \text {, }
$$


and inserting the result $\partial \beta / \partial y=0$ into Eq. (4.26) it follows that

$$
\frac{\partial^{2} \beta}{\partial t \partial y}=0
$$

The result $\partial \beta / \partial y=0$ means that $A=D$ from Eq. (4.28) and $C=B$ from Eq. (4.25). Hence, the set of equations from Eqs. (4.23) to (4.27) simplify for $\partial \beta / \partial y=0$,

$$
\begin{aligned}
& \mu=\beta^{2}(D-p)+\frac{D}{3}=\beta^{2}(A-p)+\frac{A}{3}, \\
& \frac{\partial}{\partial x}\left[\frac{\partial \beta}{\partial t}+\frac{1}{2} \frac{\partial}{\partial x}\left(\beta^{2}\right)\right]=-64 \pi B=-64 \pi C .
\end{aligned}
$$

From the result $\partial^{2} \beta / \partial x \partial y=0$ of Eq. (4.30) the set of equations (4.23)-(4.28) is recovered. Therefore, Eqs. (4.30) show that this case separates itself in two conditions, either $\partial \beta / \partial x=0$ or $\partial \beta / \partial y=0$. Next we analyze both conditions.

Case 1a: $\left[\frac{\partial \boldsymbol{\beta}}{\partial z}=\mathbf{0}\right.$ and $\left.\frac{\partial \boldsymbol{\beta}}{\partial x}=\mathbf{0}\right]$ Setting Eq. (4.27) equal to zero, then $B=-C$, and the set of equations (4.23)-(4.28) simplify to

$$
\begin{aligned}
\mu & =\beta^{2}(2 D-A-p)+\frac{A}{3}, \\
B & =-C=\frac{1}{3} A, \\
\left(\frac{\partial \beta}{\partial y}\right)^{2} & =32 \pi C, \\
\frac{\partial^{2} \beta}{\partial y^{2}} & =16 \pi \beta(A-D) .
\end{aligned}
$$

For this case, there is an equation of state given by Eq. (4.34), $\beta$ is a function of time and $y$ coordinates and must be found by solving both Eqs. (4.36) and (4.37) in terms of the pressures $A, C$ and $D$. Note that Eq. (4.35) relates the pressures $A, B$ and $C$. The EMT for this case may be written as follows:

$T_{\alpha \sigma}=\left(\begin{array}{cccc}\beta^{2}(2 D-A)+A / 3 & -\beta D & 0 & 0 \\ -\beta D & A & 0 & 0 \\ 0 & 0 & A / 3 & 0 \\ 0 & 0 & 0 & -A / 3\end{array}\right)$

One should also note that for the $T_{00}$ component from Eq. (4.38) to be of positive value the following inequality must hold:

$$
\beta^{2}>\frac{A}{3(A-2 D)}
$$

Case $1 \mathrm{~b}:\left[\frac{\partial \boldsymbol{\beta}}{\partial z}=\mathbf{0}\right.$ and $\left.\frac{\partial \boldsymbol{\beta}}{\partial \boldsymbol{y}}=\mathbf{0}\right]$ Since Eq. (4.25) is equal to zero, then $B=C$. Similarly for Eq. (4.28) it is clear that $A=D$. Consequently, the set of equations (4.23)-(4.28) simplify to

$$
\begin{aligned}
& \mu=\beta^{2}(2 D-A-p)+\frac{A}{3}, \\
& B=C+\frac{2}{3} A, \\
& B=C, \\
& A=D, \\
& \frac{\partial}{\partial x}\left[\frac{\partial \beta}{\partial t}+\frac{1}{2} \frac{\partial}{\partial x}\left(\beta^{2}\right)\right]=-64 \pi B .
\end{aligned}
$$

But from Eq. (4.25) we have $A=0$ because $B=C$. So, from Eq. (4.32) we have $\mu=-\beta^{2} p$, and one is left with a non-homogeneous Burgers equation. This case reduces the above set of equations to

$$
\begin{aligned}
& \mu=-\beta^{2} p, \\
& B=C, \\
& \frac{\partial}{\partial x}\left[\frac{\partial \beta}{\partial t}+\frac{1}{2} \frac{\partial}{\partial x}\left(\beta^{2}\right)\right]=-64 \pi B .
\end{aligned}
$$

Equation (4.45) represents an equation of state between matter density $\mu$ and the pressure $p$. The pressures $B$ and $C$ are functions of the spacetime coordinates $(t, x, y, z)$ and from Eq. (4.46) they are equal. Equation (4.47) is a non-homogeneous Burgers equation, since its right hand side cannot be readily integrated. It might mean that there is no conservation law that can describe the warp drive for the PPF EMT. The only possible way leading to conservation law is for $B$ being a constant, which then allows a straightforward integration. However, since this is not the case, namely, all the pressures from the EMT are not, necessarily, constant functions, it is necessary to determine these functions through the boundary conditions. The EMT for Case $1 \mathrm{~b}$ case then yields

$T_{\alpha \sigma}=\left(\begin{array}{llll}0 & 0 & 0 & 0 \\ 0 & 0 & 0 & 0 \\ 0 & 0 & B & 0 \\ 0 & 0 & 0 & B\end{array}\right)$.

The only non-vanishing components of the PPF EMT above are $T_{22}$ and $T_{33}$. This case recovers 
the perfect fluid Case $1 \mathrm{~b}$, that is, the dust EMTs when one chooses $p=B=0$. For $B \neq 0$, one would have to solve Eq. (4.47) to determine how the bubble moves in this type of fluid spacetime. Again, a negative matter density emerges from the Einstein equation solutions for this specific choice of EMT.

Nevertheless, this is a rather peculiar EMT, since the $T_{00}$ component is zero, but the equation of state (4.45) remains and only two diagonal terms are not zero. In addition, it is contradictory with the perfect fluid solution because the PPF reduces to the perfect fluid under the condition

$p=A=B=C=D$,

but in this solution $A=0$, but $B \neq 0$. So, we discard this case as unphysical.

Case 2: $\left.\underset{\text { plify to }}{\left[\frac{\partial \boldsymbol{\beta}}{\partial \boldsymbol{y}}=\mathbf{0}\right.}\right]$ The set of equations (4.13)-(4.22) sim-

$$
\begin{aligned}
& \mu=\beta^{2}(2 D-A-p)+\frac{A}{3} \\
& \frac{\partial^{2} \beta}{\partial x \partial z}=0 \\
& \left(\frac{\partial \beta}{\partial z}\right)^{2}=-\frac{32}{3} \pi A=-16 \pi(B-C) \\
& 2 \frac{\partial \beta}{\partial z} \frac{\partial \beta}{\partial x}+\frac{\partial^{2} \beta}{\partial t \partial z}=0 \\
& \frac{\partial}{\partial x}\left[\frac{\partial \beta}{\partial t}+\frac{1}{2} \frac{\partial}{\partial x}\left(\beta^{2}\right)\right]=-32 \pi(B+C) \\
& \frac{\partial^{2} \beta}{\partial z^{2}}=16 \pi \beta(A-D)
\end{aligned}
$$

Equation (4.52) straightforwardly implies the following relationship between pressures $A, B$ and C:

$B=C+\frac{2}{3} A$

Equations (4.51) and (4.52) indicate that $A$ and $B-C$ do not depend on the $x$-coordinate. In addition, for real solutions $A$ must be negative or zero. Finally, Eq. (4.51) shows that this case also unfolds in two subcases: $\partial \beta / \partial z=0$ and/or $\partial \beta / \partial x=0$.

Case 2a: $\left[\frac{\partial \beta}{\partial y}=\mathbf{0}\right.$ and $\left.\frac{\partial \beta}{\partial x}=\mathbf{0}\right]$ Clearly $B=-C$ as a consequence of Eq. (4.54). Then the set of equations (4.50)-(4.55) simplify to

$$
\mu=\beta^{2}(2 D-A-p)+\frac{A}{3},
$$

$$
\begin{gathered}
B=-C=\frac{A}{3}, \\
\left(\frac{\partial \beta}{\partial z}\right)^{2}=32 \pi C, \\
\frac{\partial^{2} \beta}{\partial z^{2}}=16 \pi \beta(A-D) .
\end{gathered}
$$

The EMT for this case is given by the following expression:

$$
T_{\alpha \sigma}=\left(\begin{array}{cccc}
\beta^{2}(2 D-A)+A / 3 & -\beta D & 0 & 0 \\
-\beta D & A & 0 & 0 \\
0 & 0 & -A / 3 & 0 \\
0 & 0 & 0 & A / 3
\end{array}\right),
$$

which is almost equal to Case 1a (Eq. (4.38)), apart from the change of signs in the components $T_{22}$ and $T_{33}$. Both have the same $T_{00}$ component and hence, the same equation of state, and the condition for $T_{00}$ to be of positive is given by

$\beta^{2}>\frac{A}{3(A-2 D)}$.

Besides, this expression determines another inequality that $T_{00}$ must obey to be positive,

$A-2 D>0$.

Case $2 \mathrm{~b}:\left[\frac{\partial \boldsymbol{\beta}}{\partial \boldsymbol{y}}=\mathbf{0}\right.$ and $\left.\frac{\partial \boldsymbol{\beta}}{\partial z}=\mathbf{0}\right]$ The set of equations (4.50) to (4.55) immediately simplify to

$$
\begin{aligned}
\mu & =\beta^{2}(2 D-A-p)+\frac{A}{3} \\
B & =C-\frac{2}{3} A \\
B & =C \\
A & =D=0 \\
\frac{\partial}{\partial x} & {\left[\frac{\partial \beta}{\partial t}+\frac{1}{2} \frac{\partial}{\partial x}\left(\beta^{2}\right)\right]=-64 \pi B=-64 \pi C . }
\end{aligned}
$$

Therefore $\mu=-\beta^{2} p$, a non-homogeneous Burgers equation is also present, and the expressions above are reduced to

$\mu=-\beta^{2} p$ 


$$
\begin{aligned}
& B=C \\
& \frac{\partial}{\partial x}\left[\frac{\partial \beta}{\partial t}+\frac{1}{2} \frac{\partial}{\partial x}\left(\beta^{2}\right)\right]=-64 \pi B,
\end{aligned}
$$

which are the same results as obtained in Case $1 \mathrm{~b}$ and the EMT, that is,

$$
T_{\alpha \sigma}=\left(\begin{array}{llll}
0 & 0 & 0 & 0 \\
0 & 0 & 0 & 0 \\
0 & 0 & B & 0 \\
0 & 0 & 0 & B
\end{array}\right)
$$

Again, because this solution cannot fulfill the requirement given by Eq. (4.49) — in fact it contradicts it since one cannot have both $A=0$ and $B \neq 0$-this solution is, similarly to Case $1 \mathrm{~b}$, dismissed as unphysical.

Table 2 summarizes the Einstein equations solutions obtained as a result of the PPF EMT in the Alcubierre warp drive metric.

\subsection{Discussion}

As in the perfect fluid situations, Cases $1 b$ and $2 b$ are the same for the PPF, producing the same equations of state, coordinate dependency for the $\beta$ function and a non-homogeneous Burgers equation. As stated above, only for a constant pressure $B$ a conservation law could possibly emerge from the Burgers equation (4.71) that is common to both cases. Otherwise this expression cannot be readily integrated unless further boundary conditions are met (see Sect. 5.3). Nevertheless, because the solutions of these two cases cannot satisfy the requirement established by Eq. (4.49) for reducing the PPF to the perfect fluid, they are dismissed as unphysical.

Regarding Cases 1a and 2a, the only physically plausible solutions remaining for the PPF EMT, they must obey the same inequalities (4.62) and (4.63) so that the $T_{00}$ component be positive in both Eqs. (4.38) and (4.61). The function $\beta$ depends on the $y$ coordinate in the former case and on the $z$ coordinate in the latter, and they, respectively, are a result of the integration of the following differential equations:

$\beta \frac{\partial \beta}{\partial y}=\frac{C}{A-D}$,

$\beta \frac{\partial \beta}{\partial z}=\frac{C}{A-D}$.

However, the quantities $A, C$ and $D$ are functions of the coordinates, thus integrating the expressions above requires further conditions, such as the ones, respectively, discussed in Sects. 5.1 and 5.2.

\subsubsection{Equations of state}

The perfect fluid solutions have equations of state given by [32, Ref.p. 14],

$p=p(\mu)=(\gamma-1) \mu, \quad \dot{\gamma}=\frac{\mathrm{d} \gamma}{\mathrm{d} t}=0$

Ordinary fluids can be approximated with $1 \leq \gamma \leq 2$, where incoherent matter, or dust, corresponds to $\gamma=1$, and radiation to $\gamma=\frac{4}{3}$.

In the analysis above we found that for the perfect fluid content solution Cases 1a and $2 \mathrm{a}$ for the warp drive metric the equation of state is given by $p=3 \mu$ (see Table 1 ), which corresponds to $\gamma=4$. However, they do not allow for simple expressions for the equation of state similar to Eq. (4.75) for the PPF solutions 1a and $2 \mathrm{a}$ (see Table 2). The PPF content solutions for Cases $1 \mathrm{~b}$ and $2 \mathrm{~b}$ were dismissed as unphysical,

\section{EMT divergence of the perfect fluid and PPF}

In this section we will investigate the associated conservation laws to the Einstein equations under a warp drive spacetime by means of the usual condition that the EMT divergence must be zero for both the perfect fluid and PPF. We shall start with the EMT for the PPF because from its very definition the perfect fluid can be recovered by setting the equality (4.49).

For the fluids discussed here, setting $T_{; \sigma}^{\alpha \sigma}=0$ in the EMT (4.1) results in the following expressions:

$$
\begin{aligned}
& -\frac{\partial \beta}{\partial x}(D+\mu)-\frac{\partial \mu}{\partial t} \\
& -\beta\left[\frac{\partial D}{\partial x}+\frac{\partial \mu}{\partial x}+\frac{\partial \beta}{\partial t}(2 p+A-3 D)\right] \\
& +\beta^{2}\left[\frac{\partial D}{\partial t}-\frac{\partial p}{\partial t}+3 \frac{\partial \beta}{\partial x}(D-p)\right] \\
& +\beta^{3}\left(\frac{\partial D}{\partial x}-\frac{\partial p}{\partial x}\right)=0, \\
& \frac{\partial A}{\partial x}+\frac{\partial \beta}{\partial t}(D-A) \\
& +\beta\left[3 \frac{\partial \beta}{\partial x}(D-A)+\frac{\partial D}{\partial t}-\frac{\partial A}{\partial t}\right] \\
& +\beta^{2}\left(\frac{\partial D}{\partial x}-\frac{\partial A}{\partial x}\right)=0, \\
& \frac{\partial B}{\partial y}+\beta \frac{\partial \beta}{\partial y}(D-A)=0 \\
& \frac{\partial C}{\partial z}+\beta \frac{\partial \beta}{\partial z}(D-A)=0 .
\end{aligned}
$$


Table 2 Summary of all solutions of the Einstein equations for the Alcubierre warp drive metric having the parametrized perfect fluid (PPF) EMT. The cases, conditions and their respective designations are the same as in Table 1

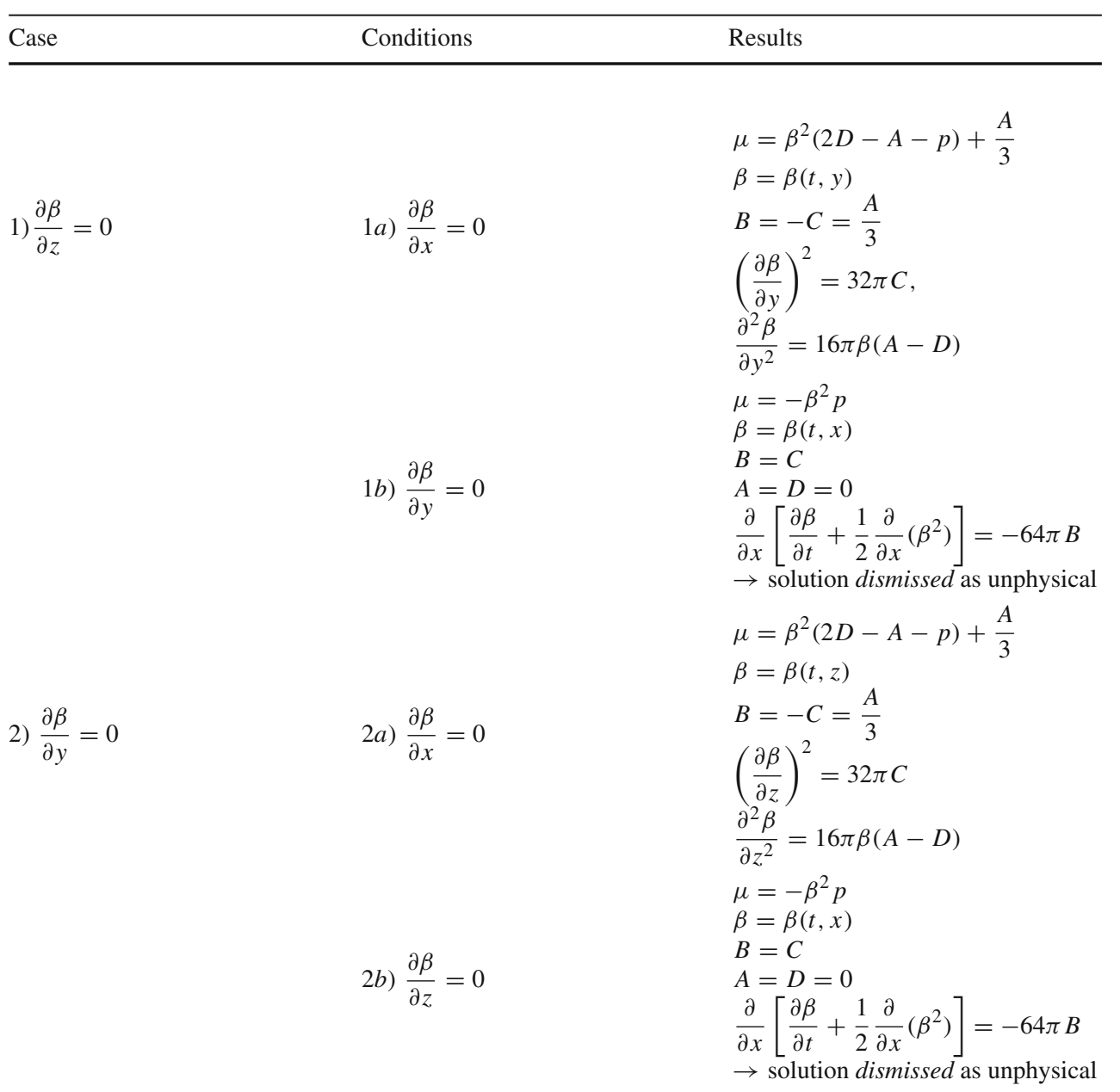

5.1 Case 1a: $\left[\frac{\partial \beta}{\partial z}=\mathbf{0}\right.$ and $\left.\frac{\partial \boldsymbol{\beta}}{\partial x}=\mathbf{0}\right]$

Equations (5.1)-(5.4) are reduced to

$$
\begin{aligned}
& -\frac{\partial \mu}{\partial t}-\beta\left[\frac{\partial D}{\partial x}+\frac{\partial \mu}{\partial x}+\frac{\partial \beta}{\partial t}(2 p+A-3 D)\right] \\
& +\beta^{2}\left(\frac{\partial D}{\partial t}-\frac{\partial p}{\partial t}\right) \\
& \quad+\beta^{3}\left(\frac{\partial D}{\partial x}-\frac{\partial p}{\partial x}\right)=0, \\
& \frac{\partial A}{\partial x}+\frac{\partial \beta}{\partial t}(D-A)+\beta\left(\frac{\partial D}{\partial t}-\frac{\partial A}{\partial t}\right) \\
& \quad+\beta^{2}\left(\frac{\partial D}{\partial x}-\frac{\partial A}{\partial x}\right)=0, \\
& \frac{\partial B}{\partial y}+\beta \frac{\partial \beta}{\partial y}(D-A)=0, \\
& \frac{\partial C}{\partial z}=0 .
\end{aligned}
$$

The results above concern the PPF. These four equations together with the five ones shown in the respective results of Table 2 mean an overdetermined system for the six unknowns $\mu, p, A, B, C, D$.

The perfect fluid is recovered by setting Eq. (4.49). Hence, Eqs. (5.5)-(5.8) become

$$
\begin{aligned}
-\frac{\partial \mu}{\partial t}-\beta\left(\frac{\partial p}{\partial x}+\frac{\partial \mu}{\partial x}\right) & =0, \\
\frac{\partial p}{\partial x} & =0, \\
\frac{\partial p}{\partial y} & =0, \\
\frac{\partial p}{\partial z} & =0,
\end{aligned}
$$

and, thus, the pressure does not depend on the spatial coordinates. In addition, Eq. (5.9) reduces to the expression

$\frac{\partial \mu}{\partial t}+\beta \frac{\partial \mu}{\partial x}=0$, 
which is the continuity equation, where $\mu$ plays the role of the fluid density and $\beta$ is the flow velocity vector field. It is worth mentioning that for a constant density the fluid has incompressible flow; then all the partial derivatives of $\beta$ in terms of the spatial coordinates vanish and the flow velocity vector field has null divergence, this being a classical fluid dynamics scenario, and the local volume dilation rate is zero.

5.2 Case 2a: $\left[\frac{\partial \beta}{\partial y}=\mathbf{0}\right.$ and $\left.\frac{\partial \beta}{\partial x}=\mathbf{0}\right]$

Equations (5.1)-(5.4) simplify to the following expressions:

$$
\begin{gathered}
-\frac{\partial \mu}{\partial t}-\beta\left[\frac{\partial D}{\partial x}+\frac{\partial \mu}{\partial x}\right. \\
\left.+\frac{\partial \beta}{\partial t}(2 p+A-3 D)\right] \\
+\beta^{2}\left(\frac{\partial D}{\partial t}-\frac{\partial p}{\partial t}\right) \\
+\beta^{3}\left(\frac{\partial D}{\partial x}-\frac{\partial p}{\partial x}\right)=0, \\
\frac{\partial A}{\partial x}+\frac{\partial \beta}{\partial t}(D-A) \\
+\beta\left(\frac{\partial D}{\partial t}-\frac{\partial A}{\partial t}\right) \\
+\beta^{2}\left(\frac{\partial D}{\partial x}-\frac{\partial A}{\partial x}\right)=0, \\
\frac{\partial B}{\partial y}=0, \\
\frac{\partial C}{\partial z}+\beta \frac{\partial \beta}{\partial z}(D-A)=0 .
\end{gathered}
$$

Assuming Eq. (4.49) the perfect fluid is recovered, resulting in the already discussed Eqs. (5.9)-(5.12) and the continuity equation (5.13).

5.3 Cases $1 \mathrm{~b}$ and $2 \mathrm{~b}:\left[\frac{\partial \beta}{\partial y}=\mathbf{0}\right.$ and $\left.\frac{\partial \beta}{\partial z}=\mathbf{0}\right]$

It follows from Table 1 that conditions $1 \mathrm{~b}$ and $2 \mathrm{~b}$ for the perfect fluid imply $\mu=p=0$, which also means $A=$ $B=C=D=0$. Then Eqs. (5.1)-(5.4) of the null EMT divergence are immediately satisfied for the perfect fluid.

Regarding the PPF, we have already seen that in these cases the solutions were dismissed as unphysical. Nonetheless, it is worth analyzing the resulting expressions to show that they lead to trivial cases or to the dust solution already studied in Ref. [19].

Table 2 shows the following solutions for the PPF EMT considering Cases $1 \mathrm{~b}$ and $2 \mathrm{~b}: A=D=0, B=C$, and $\mu=$ $-\beta^{2} p$. Hence, Eqs. (5.1)-(5.4) are reduced to the following expressions:

$$
\begin{gathered}
-\frac{\partial \mu}{\partial t}-\beta\left(\frac{\partial \mu}{\partial x}+2 p \frac{\partial \beta}{\partial t}\right) \\
-\beta^{2} \frac{\partial p}{\partial t}-\beta^{3} \frac{\partial p}{\partial x}=0, \\
\frac{\partial B}{\partial y}=0 \\
\frac{\partial C}{\partial z}=\frac{\partial B}{\partial z}=0 .
\end{gathered}
$$

Equations (5.19) and (5.20) imply that $B$ does not depend on both the $y$ and the $z$ coordinates. Inserting the state equation $\mu=-\beta^{2} p$ (see Table 2) into Eq. (5.18) yields

$p \beta^{2} \frac{\partial \beta}{\partial x}=0$.

This expression can be separated in three subcases: $\beta=0$, $p=0, \partial \beta / \partial x=0$. According to Eq. (4.71) the last subcase implies $B=0$ and, hence, there is no Burgers equation. Let us discuss below the consequences of each of these subcases.

\subsubsection{Subcase $[\beta=0]$}

This solution reduces the warp drive metric (2.6) into a Minkowski flat spacetime. There is no warp bubble and, therefore, no warp drive.

\subsubsection{Subcase $[B=0]$}

Remembering Eqs. (4.48) and (4.72) this means that all EMT components are zero, even though the equation of state $\mu=$ $-\beta^{2} p$ remains. But, since there is no EMT, both $\mu$ and $p$ must vanish, hence this case reduces itself to the dust solution [19].

\subsubsection{Subcase $[p=0]$}

Considering the equation of state $\mu=-\beta^{2} p$, then the matter density is also zero. The EMT for the PPF assumes the form given by Eq. (4.72), which implies pressure without matter density, an unphysical situation that should either be dismissed or assumed to be just the dust solution.

\section{Energy conditions}

The energy conditions are the well-known inequalities discussed by Hawking and Ellis [23], which may be applied to the matter content in physical systems as boundary conditions and to test if the energy of such systems follows positive constraint values. 
This section aims at obtaining these conditions for the Alcubierre warp drive geometry considering the previously discussed EMTs for both the perfect fluid and the PPF. Our focus will be on the main classical inequalities, namely, the weak, dominant, strong and null energy conditions. Our analysis starts with the PPF EMT defined in Eq. (4.1) in order to constrain its quantities so that the inequalities are satisfied for each of the four just named conditions. Then the same analysis for the perfect fluid is performed by reducing the results to the choice set by Eq. (4.49), but considering only the physically plausible cases.

\subsection{Weak energy condition (WEC)}

The WEC requires that at each point of the spacetime the EMT must obey the following inequality:

$T_{\alpha \sigma} u^{\alpha} u^{\sigma} \geq 0$.

This is valid for any timelike vector $\mathbf{u}\left(u_{\alpha} u^{\alpha}<0\right)$ as well as for any null vector $\mathbf{k}\left(k_{\alpha} k^{\alpha}=0\right.$; see Sect. 6.4). For an observer with a unit tangent vector $\mathbf{v}$ at some point of the spacetime, the local energy density measured by any observer is non-negative [23]. Considering the Eulerian (normal) observers from [1] with four-velocity given by the expressions

$u^{\alpha}=(1, \beta, 0,0), \quad u_{\alpha}=(-1,0,0,0)$,

and the EMT, given by Eq. (4.1), computing the expression $T_{\alpha \sigma} u^{\alpha} u^{\sigma}$ for the WEC yields

$$
\begin{aligned}
T_{\alpha \sigma} u^{\alpha} u^{\sigma} & =T_{00} u^{0} u^{0}+2 T_{01} u^{0} u^{1}+T_{11} u^{1} u^{1} \\
& =\mu+\beta^{2}(p-2 D+A) .
\end{aligned}
$$

Let us now calculate the WEC for all perfect fluid and PPF Einstein equations solutions obtained in the previous sections as referred to in Table 2.

\subsubsection{Cases $1 a$ and $2 a$}

Let us start by considering the PPF. Substituting the equation of state (4.12) into Eq. (6.3) yields

$T_{\alpha \sigma} u^{\alpha} u^{\sigma}=\frac{A}{3} \geq 0$.

So, the pressure $A$ must positive. Considering that both cases led to Eq. (4.58), the pressure $C$ must be negative. Besides, for the state equation (4.12) the density $\mu$ becomes positive if

$A+p \leq 2 D$.
This inequality implies that it is possible for the fluid density to account for both negative and positive values in a local way depending on how the momentum and pressure components relate to each other in the spacetime.

Remembering Eq. (4.49), the resulting inequality (6.4) indicates that the WEC is satisfied with the perfect fluid EMT for a positive pressure $p$. Considering Eqs. (3.30) and (3.31) that would mean a complex solution for $\beta$, a possibility already envisaged in Sect. 3.2. Note that this is a general result for the perfect fluid with a cross term solution.

\subsubsection{Cases $1 b$ and $2 b$}

It has been shown above that in these two cases the PPF solutions result in $A=D=0$ and $\mu=-\beta^{2} p$, which substituted in Eq. (6.3) yield $T_{\alpha \sigma} u^{\alpha} u^{\sigma}=0$. Hence, the WEC is not violated for the PPF.

For the perfect fluid, Table 1 shows that in these two cases the perfect fluid solutions reduce to the dust content solution for the warp drive metric, which trivially satisfies the WEC [19, Ref.p. 6].

\subsection{Dominant energy condition (DEC)}

The DEC states that for every timelike vector $u_{a}$ the following inequality must be satisfied:

$T^{\alpha \beta} u_{\alpha} u_{\beta} \geq 0$, and $F^{\alpha} F_{\alpha} \leq 0$,

where $F^{\alpha}=T^{\alpha \beta} u_{\beta}$ is a non-spacelike vector. This condition means that for any observer the local energy density is nonnegative and the local energy flow vector is non-spacelike. In any orthonormal basis the energy dominates the other components of the EMT,

$T^{00} \geq\left|T^{a b}\right|$, for each $a, b$.

Hawking and Ellis [23] suggested that this condition must hold for all known forms of matter and that it should be the case in all situations.

Evaluating this condition for PPF, the first DEC inequality is given by the following expression:

$T^{\alpha \beta} u_{\alpha} u_{\beta}=T^{00}=\beta^{2}(A-2 D+p)+\mu \geq 0$,

whereas the second inequality $F^{\alpha} F_{\alpha} \leq 0$ yields

$$
\begin{aligned}
\left(T^{\alpha \beta} u_{\beta}\right)\left(T_{\alpha \beta} u^{\beta}\right)= & -\mu^{2}-A^{2} \beta^{4}-\beta^{4} p^{2} \\
& +A^{2} \beta^{2}-\left(4 \beta^{4}-\beta^{2}\right) D^{2} \\
& +2\left(2 A \beta^{4}-A \beta^{2}\right) D
\end{aligned}
$$




$$
\begin{aligned}
& -2\left(A \beta^{2}-2 \beta^{2} D\right) \mu \\
& -2\left(A \beta^{4}-2 \beta^{4} D+\beta^{2} \mu\right) p \leq 0 .
\end{aligned}
$$

As before, let us analyze next the solutions of the Einstein equations considering each group of subcases as referred to in Table 2.

\subsubsection{Cases $1 a$ and $2 a$}

The two important results for these two cases are Eqs. (4.57) and (4.58). Substituting them into the two DEC inequalities above results in the following expressions:

$$
\begin{aligned}
& T^{\alpha \beta} u_{\alpha} u_{\beta}=\frac{A}{3} \geq 0, \\
& \left(T^{\alpha \beta} u_{\beta}\right)\left(T_{\alpha \beta} u^{\beta}\right) \\
& \quad=\left[(A-D) \beta+\frac{A}{3}\right]\left[(A-D) \beta-\frac{A}{3}\right] \leq 0 .
\end{aligned}
$$

Equation (6.10) is equal to Eq. (6.4), so this dominant energy condition is the same as the weak one. Equation (6.11) separates in two inequalities,

$$
\begin{aligned}
& -\frac{A}{3(D-A)} \leq \beta \leq \frac{A}{3(D-A)}, \\
& -\frac{A}{3(A-D)} \leq \beta \leq \frac{A}{3(A-D)},
\end{aligned}
$$

which can be rewritten as

$$
|\beta| \leq \frac{1}{3}\left|\frac{A}{D-A}\right|
$$

In addition to the inequalities above, Eq. (6.11) also leads to the following solutions:

$\beta= \pm \frac{A}{3(A-D)}$.

Equation (6.14) shows that $\beta$ is upper bounded, but since both $A$ and $D$ have no constraints, $\beta$ can be greater than unity. Remembering that $\beta=v_{s}(t) f\left[r_{s}(t)\right]$, the dominant energy condition is not violated for cases where the apparent warp bubble speed is greater than the speed of light. This result also depends on the relation between the pressure component $A$ and the momentum component $D$ as can be seen in Eqs. (6.12) and (6.13). As an example suppose that $A=D+1$ in Eq. (6.12), so that

$$
-\frac{D+1}{3} \leq v_{s}(t) f\left[r_{s}(t)\right] \leq \frac{D+1}{3} .
$$

The symmetry on the negative and positive values that $\beta$ may assume means the direction in which the warp bubble is moving on the $x$-axis on the spatial hyper surface. Note that, depending on the value of $D$, the warp bubble may assume the speed $v_{s}(t)$ greater than the speed of light.

Regarding the perfect fluid, choosing as in Eq. (4.49) the $\mathrm{DEC}$ is given by the following expressions:

$$
\begin{aligned}
T^{\alpha \beta} u_{\alpha} u_{\beta} & =\frac{p}{3} \geq 0, \\
\left(T^{\alpha \beta} u_{\beta}\right)\left(T_{\alpha \beta} u^{\beta}\right) & =p^{2} \geq 0,
\end{aligned}
$$

which means that for the DEC to be satisfied a positively valued matter density is enough (see Table 1), although this also means a complex result for $\beta$ (see Sect. 3.2).

\subsubsection{Cases $1 b$ and $2 b$}

In these cases the PPF solutions, although considered unphysical, produced $B=C, A=D=0$ and $\mu=-\beta^{2} p$ (see Table 2). So, as shown by the following expressions the DEC is immediately satisfied:

$$
\begin{aligned}
& T^{\alpha \beta} u_{\alpha} u_{\beta}=T^{00}=0, \\
& \left(T^{\alpha \beta} u_{\beta}\right)\left(T_{\alpha \beta} u^{\beta}\right)=0 .
\end{aligned}
$$

The perfect fluid is the dust solution for both cases (see Table 1 ), which is a vacuum solution and satisfies the inequalities above trivially.

\subsection{Strong energy condition (SEC)}

For any timelike vector $u^{\alpha}$ the EMT must obey the following inequality for the SEC to be valid:

$$
\left(T_{\alpha \beta}-\frac{1}{2} T g_{\alpha \beta}\right) u^{\alpha} u^{\beta} \geq 0 .
$$

This requirement is stronger than the WEC and only makes sense in a general relativistic framework because this theory is governed by the Einstein equations. These conditions imply that gravity is always attractive.

To obtain an expression for the SEC let us start with the scalar $T=g^{\alpha \beta} T_{\alpha \beta}$, where $g^{\alpha \beta}$ is given by the Alcubierre warp drive metric and $T_{\alpha \beta}$ is the EMT for the PPF (Eq. (4.1)). The result is

$T=g^{\alpha \beta} T_{\alpha \beta}=-\mu+A+B+C+\beta^{2}(2 D-p-A)$.

Substituting the equation of state (4.12) into the expression above yields

$T=\frac{2}{3} A+B+C$. 
As shown in Sect. 6.1 above, Eq. (4.12) implies that $T_{\alpha \sigma} u^{\alpha} u^{\sigma}=A / 3$ for $u^{\sigma}=(1, \beta, 0,0)$. Inasmuch as $g_{\alpha \sigma} u^{\alpha} u^{\sigma}=-1$, the SEC are given by the following expression:

$$
\left(T_{\alpha \sigma}-\frac{1}{2} T g_{\alpha \sigma}\right) u^{\alpha} u^{\sigma}=\frac{2 A}{3}+\frac{(B+C)}{2} \geq 0
$$

The particular cases, as summarized in Table 2, can now be discussed.

\subsubsection{Cases $1 a$ and $2 a$}

These cases resulted in the constraint $B=-C=A / 3$. Equation (6.24) may then be rewritten in the form

$$
\left(T_{\alpha \sigma}-\frac{1}{2} T g_{\alpha \sigma}\right) u^{\alpha} u^{\sigma}=\frac{2 A}{3}=2 B \geq 0 .
$$

Clearly, for the SEC to be satisfied it is only necessary that the pressure component $A$ must be non-negative.

Regarding the perfect fluid its respective SEC reads

$$
\left(T_{\alpha \sigma}-\frac{1}{2} T g_{\alpha \sigma}\right) u^{\alpha} u^{\sigma}=\frac{2 p}{3} \geq 0
$$

So, the SEC can be satisfied by the perfect fluid in these subcases by requiring a positive pressure $p$.

\subsubsection{Cases $1 b$ and $2 b$}

In these cases the PPF solutions stated that $A=D=0$ and $B=C$. Hence, the SEC is satisfied under the constraint

$$
\left(T_{\alpha \sigma}-\frac{1}{2} T g_{\alpha \sigma}\right) u^{\alpha} u^{\sigma}=B \geq 0
$$

despite providing unphysical solutions. The perfect fluid solutions are just the dust solution, that is, a vacuum solution [19], thus satisfying the SEC trivially.

\subsection{Null energy condition (NEC)}

The SEC and WEC are satisfied in the limit of the null observers with four-velocities $\mathbf{k}$. To satisfy the NEC the EMT must obey the inequality

$T_{\alpha \sigma} k^{\alpha} k^{\sigma} \geq 0$, for any null vector $k^{\alpha}$.

To calculate the NEC let us consider the following null vector:

$k^{\alpha}=(a, b, 0,0)$ where the relation between $a$ and $b$ can be obtained by imposing the condition

$k_{\alpha} k^{\alpha}=0$,

which leads to

$a^{2} \beta^{2}-2 a b \beta-a^{2}+b^{2}=0$,

whose roots for $a / b$ yield

$\frac{a}{b}=\frac{1}{\beta+1}$ and $\frac{a}{b}=\frac{1}{\beta-1}$.

Considering the results above, the NEC may be written as follows:

$T_{\alpha \sigma} k^{\alpha} k^{\sigma}=T_{00} k^{0} k^{0}+2 T_{01} k^{0} k^{1}+T_{11} k^{1} k^{1}$,

where $T_{\alpha \sigma}$ is the PPF EMT of Eq. (4.1). So, the null condition is given by the following expression:

$T_{\alpha \sigma} k^{\alpha} k^{\sigma}=a^{2} \beta^{2} p-2 a b \beta D+b^{2} A+a^{2} \mu \geq 0$.

Substituting the two roots in Eqs. (6.32) into Eq. (6.34) results in the expressions

$$
\begin{aligned}
& \frac{b^{2}}{(\beta+1)^{2}}\left[\mu-\beta^{2}(2 D-A-p)\right. \\
& +2 \beta(A-D)+A] \geq 0, \\
& \frac{b^{2}}{(\beta-1)^{2}}\left[\mu-\beta^{2}(2 D-A-p)\right. \\
& -2 \beta(A-D)+A] \geq 0 .
\end{aligned}
$$

Next, as before, we shall proceed to investigate first Cases $1 \mathrm{a}$ and $2 \mathrm{a}$ and then $1 \mathrm{~b}$ and $2 \mathrm{~b}$, as defined in Tables 1 and 2, for the PPF and the perfect fluid.

\subsubsection{Cases $1 a$ and $2 a$}

Considering the PPF first, the equation of state (4.57) is a solution of both cases (see Table 2), so the resulting NEC given by Eqs. (6.35) and (6.36) simplify to the following expressions:

$\frac{b^{2}}{(\beta+1)^{2}}\left[\frac{4 A}{3}+2 \beta(A-D)\right] \geq 0$,
$\frac{b^{2}}{(\beta-1)^{2}}\left[\frac{4 A}{3}-2 \beta(A-D)\right] \geq 0$,

whose solutions for $\beta$ yield the NEC for the PPF EMT:

$\beta \geq \frac{2}{3} \frac{A}{D-A}$ and $\beta \neq-1$ 
$\beta \leq \frac{2}{3} \frac{A}{A-D}$ and $\beta \neq 1$.

Remembering that $\beta=v_{s}(t) f\left(r_{s}\right)$, then Eq. (6.39) states that the warp bubble cannot assume the negative sign of the speed of light, but it may assume values above it. To verify that, we have to choose $A=D-1>2 / 3$ and $\beta>1$. Equation (6.40) states that $\beta$ cannot assume the exact value of the speed of light and it is bounded by a superior value which can be greater than the speed of light. To have this, one just have to choose for example $A=D+1>2 / 3$.

For the perfect fluid, according to Eq. (4.49) all pressures are equal. Hence, substituting the two values for $a / b$ found in Eq. (6.34) into Eqs. (6.35) and (6.36) they then simplify to the expressions

$$
\begin{aligned}
& \frac{b^{2}}{(\beta+1)^{2}}(\mu+p) \geq 0, \\
& \frac{b^{2}}{(\beta-1)^{2}}(\mu+p) \geq 0 .
\end{aligned}
$$

These results mean that the NEC is satisfied for the perfect fluid provided that

$\mu+p \geq 0$

Remembering that $p=3 \mu$ for the perfect fluid in these two cases, then the NEC is fulfilled if the matter density does not have negative values.

\subsubsection{Cases $1 b$ and $2 b$}

These cases, although set as unphysical, we already concluded that the PPF relates its pressures by the following expressions: $A=D=0$ and $B=C$. Therefore, Eqs. (6.35) and (6.36) yield

$$
\begin{aligned}
& \frac{b^{2}}{(\beta+1)^{2}}\left(\mu+\beta^{2} p\right) \geq 0, \\
& \frac{b^{2}}{(\beta-1)^{2}}\left(\mu+\beta^{2} p\right) \geq 0 .
\end{aligned}
$$

So, the NEC establishes that $\mu+\beta^{2} p \geq 0$, which is readily satisfied since in these cases we already concluded that $\mu=$ $-\beta^{2} p$, provided that $\beta \neq-1$ for the former and $\beta \neq 1$ for the latter. Regarding the perfect fluid, these cases reduce to the dust solution studied in [19], which is a vacuum solution and, therefore, immediately satisfies the NEC.

Table 3 summarizes the results of all energy conditions for the PPF and perfect fluid EMTs with the Alcubierre warp drive metric.

\section{Further discussions}

This section discusses some points, and raises others, all related to the physics of the warp drive as suggested by the results presented in the previous sections. It aims at offering some thoughts that may be important in fostering further understanding on how a superluminal travel can be achieved.

\subsection{Regulating function and the Burgers equation}

The regulating function (2.8) describes the shape of the warp bubble, but it is not uniquely determined. However, the integration of the Einstein equations in both the perfect fluid and the PPF led to the appearance of generic functions in the dynamic equations which may end up connected to the regulating function, a situation that adds to its nonuniqueness. This means that physically feasible superluminal speeds will require the specification of these generic functions, possibly by boundary conditions. We shall show below an example of this situation using the dust solution.

Considering Eqs. (2.3) and (2.10) the partial derivative of the Burgers equation (3.19) yields

$$
\frac{\partial \beta}{\partial t}=f \frac{\mathrm{d}^{2} x_{s}}{\mathrm{~d} t^{2}}+v_{s} \frac{\partial f}{\partial r_{s}} \frac{\partial r_{s}}{\partial t},
$$

where the simplified notation $f=f\left[r_{s}(t)\right]$ and $v_{s}=v_{s}(t)$ was adopted. Since

$\frac{\partial \beta}{\partial x}=v_{s} \frac{\partial f}{\partial r_{s}} \frac{\partial r_{s}}{\partial x}$,

the Burgers equation becomes

$f \frac{\mathrm{d}^{2} x_{s}}{\mathrm{~d} t^{2}}+v_{s} \frac{\partial f}{\partial r_{s}} \frac{\partial r_{s}}{\partial t}+v_{s}^{2} f \frac{\partial f}{\partial r_{s}} \frac{\partial r_{s}}{\partial x}=h(t)$.

The partial derivative of Eq. (2.8) may be written as follows:

$$
\begin{aligned}
\frac{\partial f}{\partial r_{s}}= & \frac{\sigma}{2 \tanh (\sigma R)}\left\{\operatorname{sech}^{2}\left[\sigma\left(r_{s}+R\right)\right]\right. \\
& \left.-\operatorname{sech}^{2}\left[\sigma\left(r_{s}-R\right)\right]\right\} .
\end{aligned}
$$

The partial derivatives of $r_{s}$ yield

$$
\begin{aligned}
\frac{\partial r_{s}}{\partial t} & = \pm \frac{\mathrm{d} x_{s}}{\mathrm{~d} t}= \pm v_{s}(t), \\
\frac{\partial r_{s}}{\partial x} & = \pm 1
\end{aligned}
$$

and remembering that in the dust case $\beta=\beta(x, t)$, then $r_{s}(t)$ is given by (see Eq. (2.9))

$r_{S}(t)=\sqrt{\left[x-x_{S}(t)\right]^{2}}=\left|x-x_{S}(t)\right|$. 
Table 3 Summary of all energy conditions results for the perfect fluid and PPF EMTs with the Alcubierre warp drive spacetime geometry

\begin{tabular}{|c|c|c|}
\hline Cases (refer to Tables 1 and 2) & Energy Conditions & Results \\
\hline \multirow[t]{4}{*}{$1 \mathrm{a}$ and $2 \mathrm{a}$} & Weak & $\begin{array}{l}\text { Perfect fluid: } p \geq 0 \\
\text { PPF: } A \geq 0\end{array}$ \\
\hline & Dominant & $\begin{array}{l}\text { Perfect Fluid: } p \geq 0 \\
\text { PPF: } A \geq 0 \text { and }|\beta| \leq \frac{1}{3}\left|\frac{A}{D-A}\right|\end{array}$ \\
\hline & Strong & $\begin{array}{l}\text { Perfect fluid : } p \geq 0 \\
\text { PPF : } A \geq 0\end{array}$ \\
\hline & Null & $\begin{array}{l}\text { Perfect fluid: } \mu+p \geq 0 \\
\text { PPF : } \beta \geq \frac{2}{3} \frac{A}{D-A} \text { and } \beta \neq-1 \\
\text { PPF : } \beta \leq \frac{2}{3} \frac{A}{A-D} \text { and } \beta \neq 1\end{array}$ \\
\hline \multirow[t]{4}{*}{$1 b$ and $2 b$} & Weak & immediately satisfied for perfect fluid and PPF. \\
\hline & Dominant & immediately satisfied for perfect fluid and PPF. \\
\hline & Strong & $\begin{array}{l}\text { Perfect fluid: immediately satisfied. } \\
\text { PPF: } B \geq 0\end{array}$ \\
\hline & Null & immediately satisfied for perfect fluid and PPF. \\
\hline
\end{tabular}

Considering the expressions above, Eq. (7.3) may be rewritten as follows:

$$
\begin{aligned}
& f \frac{\mathrm{d}^{2} x_{s}}{\mathrm{~d} t^{2}} \pm v_{s}^{2} \frac{\sigma F}{2 \tanh (\sigma R)} \\
& \quad \pm v_{s}^{2} f \frac{\sigma F}{2 \tanh (\sigma R)}=h(t)
\end{aligned}
$$

where

$$
\begin{aligned}
F\left(r_{s}\right) \equiv & \operatorname{sech}^{2}\left[\sigma\left(r_{s}+R\right)\right] \\
& -\operatorname{sech}^{2}\left[\sigma\left(r_{s}-R\right)\right] .
\end{aligned}
$$

Remembering that the regulating function $f\left[r_{s}(t)\right]$, as defined by Eq. (2.8), can be approximated by a top hat function when $\sigma \gg R$ (see Ref. [19], §2.1), in this limit Eq. (7.8) takes the following form inside the bubble:

$$
\frac{\mathrm{d}^{2} x_{s}}{\mathrm{~d} t^{2}}=h(t),
$$

where $f=1$. Outside the bubble $f=0$ and then $h(t)=0$, which means plane shock waves described by the inviscid Burgers equation [19].

Hence, the nonuniqueness of the shift vector $\beta=$ $v_{s}\left(r_{s}\right) f\left[r_{s}(t)\right]$ arises not only from the fact that the function $h(t)$ is arbitrary, but also because the regulating function $f\left[r_{s}(t)\right]$ only requires a top hat behavior with null values outside the bubble. So, any well-behaved function that respects such constraints may be part of a solution of the Burgers equation. Moreover, from the energy conditions calculated for the PPF, as summarized in Table 3 , one can see that $\beta$ plays a fundamental role in Cases $1 \mathrm{a}$ and $2 \mathrm{a}$ for the null and dominant energy conditions to be satisfied, which adds further physical constraints to its behavior. So, it is clear that the nonlinearity of the Einstein equations imply that the generic functions appearing in their integration become entangled with the regulating function in a non-trivial manner.

To extend this analysis to the perfect fluid and PPF contents requires further constraints on the shift vector, something which at this stage would be done in an entirely arbitrary manner. Perhaps in the future this can be done under more physically plausible reasoning.

\subsection{Anisotropic fluids}

The PPF proposed in Sect. 4 aimed at offering an alternative EMT for solving the Einstein equations endowed with the warp drive metric. As we shall see below, the PPF can actually be seen as an anisotropic fluid with heat flux [33].

In general relativity the energy-momentum tensor $T_{\mu \nu}$ represents the source of energy and momentum, where $T_{00}$ is the flow of energy across a surface of constant time (energy density), $T_{0 i}$ is the energy flux across a surface in the $i$ direction (constant $\left.x^{i}\right), T_{i 0}$ is the momentum density and $T_{i j}$ is the momentum flux. If we choose a comoving frame of reference that moves with the same velocity as the fluid this means that particles in this fluid will have zero velocity and the flux of energy will be only through the flux of heat, and the momentum flux will be via some sort of dissipative phe- 
nomena such as viscosity, thermal radiation or even some sort of electromagnetic type of radiation.

The general stress-energy tensor of a relativistic fluid can be written in the form $[33,34]$

$T^{\alpha \beta}=\mu u^{\alpha} u^{\beta}+p h^{\alpha \beta}+u^{\alpha} q^{\beta}+u^{\beta} q^{\alpha}+\pi^{\alpha \beta}$,

where

$h_{\alpha \beta}=g_{\alpha \beta}+u_{a} u_{b}$

projects tensors onto hypersurfaces orthogonal to $u^{\alpha}, \mu$ is the matter density, $p$ is the fluid static pressure, $q^{\alpha}$ is the heat flux vector and $\pi^{\alpha \beta}$ is the viscous shear tensor. The world lines of the fluid elements are the integral curves of the four-velocity vector $u^{\alpha}$. The heat flux vector and viscous shear tensor are transverse to the world lines, that is,

$q_{a} u^{a}=0, \quad \pi_{a b} u^{b}=0$.

In terms of coordinates we can write the energy-momentum tensor for a general fluid as

$T_{\alpha \beta}=\left(\begin{array}{cc}\varepsilon & q_{a} \\ q_{b} & \pi_{a b}\end{array}\right)$,

where $q_{a}$ is the three-vector heat flux vector, $\varepsilon$ is a scalar function and $\pi_{a b}$ is a three-by-three matrix viscous stress tensor, which is symmetric and traceless. Both $q_{a}$ and $\pi_{a b}$ have, respectively, three and five linearly independent components. Together with the density $\mu$ and the static pressure $p$, this makes a total of ten linearly independent components, which is the number of linearly independent components in a four-dimensional symmetric rank two tensor. We noticed that the Einstein tensor components are highly nonlinear for the warp drive metric and the off-diagonal terms require those free parameters for a non-overdetermined solution. For a noncurved metric, i.e., the Minkowski metric $\eta_{\alpha \beta}$, the energymomentum tensor for a perfect fluid with anisotropic pressures can be written as

$T_{\alpha \beta}=\left(\begin{array}{cccc}\mu & 0 & 0 & 0 \\ 0 & p_{x} & 0 & 0 \\ 0 & 0 & p_{y} & 0 \\ 0 & 0 & 0 & p_{z}\end{array}\right)$.

Isotropic static pressure means that $p_{x}=p_{y}=p_{z}=p$. The perfect fluid has no heat flux or dissipative phenomena, then $\left(q^{\alpha}=0, \pi^{\alpha \beta}=0\right)$. This special case with dust content is the well-known EMT, that is,

$T^{\alpha \beta}=\mu u^{\alpha} u^{\beta}+p h^{\alpha \beta}=(\mu+p) u^{\alpha} u^{\beta}+p g^{\alpha \beta}$.

The PPF proposed in Sect. 4 has the matrix form given by Eq. (4.1), which may be broken down as the sum of a perfect fluid in a warp drive background as given by Eq. (3.2), and a dissipative fluid with the heat flux four-vector given by $q_{\alpha}=-\frac{1}{2}\left(q_{0}, q_{1}, 0,0\right)$. Hence

$q_{\alpha} u_{\beta}+q_{\beta} u_{\alpha}=\left(\begin{array}{cccc}q_{0} & q_{1} & 0 & 0 \\ q_{1} & 0 & 0 & 0 \\ 0 & 0 & 0 & 0 \\ 0 & 0 & 0 & 0\end{array}\right)$

since the four-velocity for the moving frame is $u_{\alpha}=$ $(-1,0,0,0)$. The isotropic term is given by

$\pi_{\alpha \beta}=\left(\begin{array}{cccc}\pi_{00} & 0 & 0 & 0 \\ 0 & \pi_{01} & 0 & 0 \\ 0 & 0 & \pi_{02} & 0 \\ 0 & 0 & 0 & \pi_{03}\end{array}\right)$

and

$\mu+\beta^{2} p \rightarrow\left(\mu+\pi_{00}+q_{0}\right)+\beta^{2} p$.

So, the four parameters of the PPF are as follows:

$$
\begin{aligned}
& A=\pi_{01}+p, \\
& B=\pi_{02}+p, \\
& C=\pi_{03}+p, \\
& D=p-\frac{q_{1}}{\beta} .
\end{aligned}
$$

The tensor $\pi_{\alpha \beta}$ must be traceless, giving us one more equation to solve for the free parameters (anisotropic pressures)

$\pi_{00}+\pi_{01}+\pi_{02}+\pi_{03}=0$.

From the above one can see that the warp drive metric endowed with the PPF allows one to make a study of fluid anisotropy coupled with possible dissipative effects that could lead to a warp drive bubble. Perfect fluids are well known to be part of solutions for the Einstein equations, this being the case of the standard FLRW cosmological model that accounts for the expansion, isotropy and spatial homogeneity of the universe. On the other hand, anisotropic imperfect fluids offer a more complex source of gravitational effects, presenting dissipative processes, shear and bulk viscous pressures, interaction between fluids, radiation processes, electromagnetic interaction and even collision between particles, charged or not. These fluids are even known to avoid the big bang singularity in cosmological models [35-39]. MacCallum [40] discussed various ways of generating anisotropy such as the presence of electromagnetic fields, the presence of viscous terms and the anisotropic stresses due to the anisotropic expansion of a cloud of collisionless particles. Another way to account for viscosity, 
heat and energy flux is the interaction of two or more fluids $[41,42]$.

\subsection{Other aspects of warp drive physics}

The points presented above regarding the physical feasibility of superluminal travel with the Alcubierre spacetime geometry by no means exhaust this discussion. Several issues remain open, with each of them deserving separate studies that are beyond the scope of this paper, since in here we focused on the basic properties of the solutions of the Einstein equations of the warp drive metric with fluid content. With respect to the open issues, one can point out the amount of mass-energy density, exotic or not, necessary for the feasibility for the warp drive in the context of both the perfect fluid and the PPF, and also quantum effects and the question of stability or instability in our solutions. These issues deserve further investigations and are the subject of ongoing research.

\section{Conclusions}

In this work we have analyzed the Einstein equations for the Alcubierre warp drive metric having as gravity source two types of energy-momentum tensors (EMT) for a fluid, namely the perfect fluid and the parametrized perfect fluid (PPF). The latter is defined by allowing the EMT pressure components of the perfect fluid to be different from one another and dependent on all coordinates.

After obtaining the components of the Einstein tensor for the warp drive metric we calculated the dynamic equations for both fluids by solving the respective Einstein equations. Solutions were found in the form of various equations of state, and, by further imposing the null divergence for the EMTs, new constraints were also found for the various variables. The weak, dominant, strong and null energy conditions were also calculated, which implied further constraints upon the free quantities for the EMTs. For the perfect fluid these were on the matter density $\mu$ and pressure $p$. For the PPF that occurred these were on the pressure components $p, A, B, C$, the matter density for the fluid $\mu$ and the momentum component $D$.

We found two main groups of solution subcases possessing different conditions for each EMT. For the perfect fluid, one solution may be interpreted as requiring that the warp bubble can only be viable with negative matter density. The alternative interpretation is that the warp bubble is possible with positive matter density, but in this case the regulating function $f(r, s)$, which shapes the warp bubble, becomes a complex function. This comes from results allowing the possibility that the function $\beta=v_{s}(t) f\left(r_{s}\right)$ may have complex solutions once the matter density is positive. Other results in both fluids reinforce our earlier finding in Ref. [19] that the warp bubble necessary for generating superluminal velocities, or warp speeds, can be interpreted as a shock wave from classical fluid dynamics theory.

We mentioned four cases arising from the solutions of the Einstein equations: 1a, 1b, 2a and 2b. However, Cases $1 \mathrm{a}$ and $2 \mathrm{a}$ are very similar, or equal, to each other, and we have the same for Cases $1 \mathrm{~b}$ and $2 \mathrm{~b}$. For this reason they were grouped together in the tables that summarize all results.

Specifically, Cases $1 \mathrm{~b}$ and $2 \mathrm{~b}$ for the perfect fluid reduced the solutions to the ones found for dust content already studied in Ref. [19], that is, a vacuum solution unable to create a warp bubble, but which connects the warp metric to the inviscid Burgers equation, also yielding $\beta$ as a function of $t$ and $x$ coordinates. The null EMT divergence is satisfied and a continuity equation was also found (Eq. (5.13)).

Cases $1 \mathrm{~b}$ and $2 \mathrm{~b}$ for the PPF resulted in an equation of state of the form $\mu=-\beta^{2} p$, the coordinate dependency of the $\beta$ function became $\beta=\beta(x, t)$ and a non-homogeneous Burgers equation (4.71) emerged. However, the pressures were constrained in such a way that this PPF EMT solution for the warp drive was dismissed as unphysical.

Cases 1a and $2 \mathrm{a}$ for the perfect fluid produced an equation of state relating pressure and matter density given by $p=3 \mu$. The $\beta$ function dependencies became $\beta=\beta(y, t)$ in the former case and $\beta=\beta(z, t)$ in the latter case, and both produced a differential equation for $\beta$ that either requires a negative density for $\beta$ to be a real valued function, or a positive matter density which then leads to a complex solution for $\beta$, which in turn leads to a complex regulating function $f\left(r_{s}\right)$ whose possible real part would then be related to a physically viable warp bubble.

Cases $1 \mathrm{a}$ and $2 \mathrm{a}$ for the PPF resulted in an equation of state relating almost all quantities, in the form $\mu=\beta^{2}(2 D-$ $A-p)+A / 3$ which is valid for both cases. The coordinate dependency on $\beta$ that occurred, respectively, resulted in $\beta=$ $\beta(y, t)$ and $\beta=\beta(z, t)$. The function $\beta$ is also governed by the first order differential equations (4.73) and (4.74), respectively.

The null EMT divergence $T^{\alpha \sigma}{ }_{; \sigma}=0$ was calculated, producing further sets of very nonlinear differential equations constraining all quantities in the PPF which could be used, in principle, to determine all pressures and matter density in this fluid. For the perfect fluid, Cases $1 \mathrm{a}$ and $2 \mathrm{a}$ are reduced to a continuity equation (5.13) including the function $\beta$, which is interpreted as playing the role of the flow velocity vector field. Cases $1 b$ and $2 b$ reduced the PPF to either the trivial condition of Minkowski flat spacetime with no warp drive, or an EMT with all components being zero, that is, a vacuum case.

It has already been seen in Ref. [19] that the Burgers equation appears to be connected to the dust solution of the warp drive metric, which is in fact a vacuum solution. Cases $1 b$ 
and $2 \mathrm{~b}$ of the perfect fluid became reduced to the dust solution, and a non-homogeneous form of the Burgers equation appears in these respective cases for the PPF, although the whole solutions in these cases were dismissed as unphysical. The solutions that presented themselves as the most plausible ones for creating warp speeds, $1 \mathrm{a}$ and $2 \mathrm{a}$ for both the perfect fluid and the PPF, do not generate a Burgers equation.

The weak, dominant, strong and null energy conditions were also studied in the context of the perfect fluid and PPF energy-momentum tensors for a warp drive metric. The resulting expressions were found to satisfy all conditions in the perfect fluid EMT. Regarding the PPF, specific expressions constraining its EMT quantities were obtained in order to satisfy these energy conditions, but they do not necessarily lead to the conclusion that negative matter density is always necessary for viable warp speeds, particularly because in the PPF the pressure $A$ must assume values equal to or greater than zero.

Summing up, the results of this paper indicate that warp speeds might be physically viable in the context of positive matter density as some solutions of the Einstein equations for both fluids keep open this possibility. Nevertheless, such a situation creates the additional issue in the perfect fluid context concerning the meaning of a possible complex regulating function in the warp metric, a result that may be interpreted as a caveat, or major stumbling block. Such a difficulty does not appear to occur in the PPF scenario, although this fluid was considered here mainly as a hypothetical model whose aim was to investigate whether or not new possibilities arise in the solutions of the Einstein field equations considering more complex energy-momentum tensors having the Alcubierre warp drive metric. On this front it seems then that the initial conclusions about the unphysical nature of the warp drive, or the impossibility of generating warp speeds, may not be not as stringent as initially thought, or, perhaps, are not valid at all.

Acknowledgements We are grateful to the referees for useful comments. E.M.C.A. thanks CNPq (Conselho Nacional de Desenvolvimento Científico e Tecnológico), Brazilian scientific support federal agency, for partial financial support, Grants numbers 406894/2018-3 and 302155/2015-5.

Data Availability Statement This manuscript has no associated data or the data will not be deposited. [Authors' comment: This a theoretical work. There is no data available and, hence, no data will be deposited.]

Open Access This article is licensed under a Creative Commons Attribution 4.0 International License, which permits use, sharing, adaptation, distribution and reproduction in any medium or format, as long as you give appropriate credit to the original author(s) and the source, provide a link to the Creative Commons licence, and indicate if changes were made. The images or other third party material in this article are included in the article's Creative Commons licence, unless indicated otherwise in a credit line to the material. If material is not included in the article's Creative Commons licence and your intended use is not permitted by statutory regulation or exceeds the permit- ted use, you will need to obtain permission directly from the copyright holder. To view a copy of this licence, visit http://creativecomm ons.org/licenses/by/4.0/.

Funded by $\mathrm{SCOAP}^{3}$.

\section{References}

1. M. Alcubierre, The warp drive: hyper-fast travel within general relativity. Class. Quantum Gravity 11, L73 (1994). arXiv:gr-qc/0009013

2. L.H. Ford, T.A. Roman, Quantum field theory constrains traversable wormhole geometries. Phys. Rev. D 53, 5496 (1996). arXiv:gr-qc/9510071

3. M.J. Pfenning, L.H. Ford, The unphysical nature of warp drive. Class. Quantum Gravity 14, 1743 (1997). arXiv:gr-qc/9702026

4. W.A. Hiscock, Quantum effects in the Alcubierre warp-drive spacetime. Class. Quantum Gravity 14, L183 (1997)

5. S.V. Krasnikov, Hyperfast interstellar travel in general relativity. Phys. Rev. D 57, 4760 (1998). arXiv:gr-qc/9511068

6. A. Everett, T.A. Roman, A superluminal subway: the Krasnikov tube. Phys. Rev. D 56, 2100 (1997). arXiv:gr-qc/9702049

7. F.S.N. Lobo, P. Crawford, Weak energy condition violation and superluminal travel, in Current Trends in Relativistic Astrophysics. Lecture Notes in Physics, vol. 617, ed. by L. FernándezJambrina, L.M. González-Romero (Springer, Berlin, 2003). arXiv:gr-qc/0204038

8. F.S.N. Lobo, P. Crawford, Weak energy condition violation and superluminal travel. Lect. Notes Phys. 617, 277 (2003). arXiv:gr-qc/0204038

9. C. Van Den Broeck, A warp drive with more reasonable total energy. Class. Quantum Gravity 16, 3973 (1999). arXiv:gr-qc/9905084

10. J. Natario, Warp drive with zero expansion. Class. Quantum Gravity 19, 1157 (2002). arXiv:gr-qc/0110086

11. F.S.N. Lobo, M. Visser, Fundamental limitations on 'warp drive' spacetimes. Class. Quantum Grav. 21, 5871 (2004). arXiv:gr-qc/0406083

12. F.S.N. Lobo, M. Visser, Linearized warp drive and the energy conditions. arXiv:gr-qc/0412065

13. H.G. White, A discussion of space-time metric engineering. Gen. Relativ. Gravit. 35, 2025 (2003)

14. H.G. White, Warp field mechanics 101. J. Br. Interplanet. Soc. 66, $242(2011)$

15. J. Lee, G. Cleaver, Effects of external radiation on an Alcubierre warp bubble. Phys. Essays 29, 201 (2016)

16. J. Lee, G. Cleaver, The inability of the White-Juday warp field interferometer to spectrally resolve spacetime distortions. Int. J. Mod. Phys. Adv. Theory Appl. 2, 35 (2017). arXiv:1407.7772

17. B. Mattingly, A. Kar, M. Gorban, W. Julius, C. Watson, M.D. Ali, A. Baas, C. Elmore, J. Lee, B. Shakerin, E. Davis, G. Cleaver, Curvature invariants for the accelerating Natario warp drive. Particles 3, 642 (2020). arXiv:2008.03366

18. B. Mattingly, A. Kar, M. Gorban, W. Julius, C.K. Watson, M. Ali, A. Baas, C. Elmore, J.S. Lee, B. Shakerin, E.W. Davis, G.B. Cleaver, Curvature invariants for the Alcubierre and Natário warp drives. Universe 7, 21 (2021). arXiv:2010.13693v2

19. O.L. Santos-Pereira, E.M.C. Abreu, M.B. Ribeiro, Dust content content solutions for the Alcubierre warp drive spacetime. Eur. Phys. J. C 80, 786 (2020). arXiv:2008.06560

20. B. Schutz, A First Course in General Relativity, 2nd edn. (Cambridge University Press, Cambridge, 2009)

21. M. Alcubierre, Introduction to 3+1 Numerical Relativity (International Series of Monographs on Physics) (Oxford University Press, Oxford, 2012) 
22. B.S. DeWitt, in General relativity: an Einstein centenary survey, ed. by S.W. Hawking, W. Israel (Cambridge University Press, Cambridge, 1980)

23. S.W. Hawking, G.F.R. Ellis, The Large Scale Structure of SpaceTime (Cambridge University Press, Cambridge, 1973)

24. L.N. Trefethen, K. Embree (editors), unpublished (2001). https:// people.maths.ox.ac.uk/trefethen/pdectb.html. Accessed 14 Oct 2018

25. L.C. Evans, Partial Differential Equations, Graduate Studies in Mathematics, vol. 19 (American Mathematical Society, Providence, 2010), p. 749

26. A.R. Forsyth, Theory of Differential Equations (University Press, Cambridge, 1906)

27. H. Bateman, Some recent researches on the motion of fluids. Mon. Weather Rev. 43, 163 (1915)

28. J.M. Burgers, A mathematical model illustrating the theory of turbulence. Adv. Appl. Mech. 1, 171 (1948)

29. T. Ceylan, B. Okutmuştur, Finite volume approximation of the relativistic Burgers equation on a Schwarzschild(anti-)de Sitter spacetime. Turk. J. Math. 41, 1027 (2017)

30. T. Ceylan, B. Okutmuştur, The relativistic Burgers equation on a de Sitter spacetime. Derivation and finite volume approximation. Int. J. Pure. Math. 2, 20 (2015)

31. P.G. LeFloch, H. Makhlof, B. Okutmuştur, Relativistic Burgers equation on curved spacetime. Derivation and finite volume approximation. arXiv: 1206.3018

32. G. Ellis, H. van Elst, Cargese lectures 1998: cosmological models. NATO Adv. Study Inst. Ser. C. Math. Phys. Sci. 541, 1-116 (1999). arXiv:gr-qc/9812046

33. C. Eckart, The thermodynamics of irreversible processes. III. Relativistic theory of the simple fluid. Phys. Rev. 58, 919 (1940)
34. O.M. Pimentel, F.D. Lora-Clavijo, G.A. Gonzalez, The energymomentum tensor for a dissipative fluid in general relativity. Gen. Relativ. Gravit. 48, 124 (2016)

35. G.L. Murphy, Big-bang model without singularities. Phys. Rev. D 8, 4231 (1973)

36. Z. Klimek, Some thermodynamic features of cosmological models with viscosity. Acta Cosmol. 2, 49 (1974)

37. M.B. Ribeiro, Cosmological models with dissipative fluids, Master's thesis, Observatório Nacional, Rio de Janeiro, 1987. https:// doi.org/10.13140/RG.2.1.1184.0724 (In Portuguese)

38. M.B. Ribeiro, A.K. Sanyal, Bianchi $\mathrm{VI}_{0}$ viscous fluid cosmology with magnetic field. J. Math. Phys. 28, 657 (1987)

39. A. Banerjee, M.B. Ribeiro, N.O. Santos, Anisotropic viscous fluid cosmological model. Astrophys. Space Sci. 136, 331 (1987)

40. M.A.H. MacCallum, In: General Relativity: An Einstein Centenary Survey, ed. S.W. Hawking, W. Israel (Cambridge University Press, 1979)

41. P.S. Letelier, Anisotropic fluids with two-perfect-fluid components. Phys. Rev. D 22, 807 (1980)

42. S.S. Bayin, Anisotropic fluid spheres in general relativity. Phys. Rev. D 26, 1262 (1982) 